\title{
Modelling of dynamical crack propagation using time-domain boundary integral equations
}

\author{
Martin G. Koller \\ Laboratoire de Géophysique Interne et Tectonophysique (URA CNRS 733), IRIGM. Université Joseph Fourier BP 53X, \\ F38041 Grenoble cedex, France \\ Marc Bonnet \\ Laboratoire de Mécanique des Solides, (centre commun X-Mines-Ponts, URA CNRS 317), Ecole Polytechnique, \\ 91128 Palaiseau cedex, France

\section{Raul Madariaga} \\ Laboratoirc de Sismologie, Institut de Physique du Globe, Université Pierre et Marie Curie, 4 place Jussieu, \\ 75005 Paris, France
}

Received 1 August 1991, Revised 1 July 1992

\begin{abstract}
We study dynamic antiplane cracks in the time domain by the boundary integral equation method (BIEM) based on the integral equation for dis cement discontinuity (or crack opening displacement, $C O D$ ) as a function of stress on the crack. This displacement discontinuity formulation present 3 the advantage, with respect to methods developed by Das and others in seismology, that it has to be solved only inside the crack. This BIEM is, however, difficult to implement numerically because of the hypersingularity of the kernel of the integral equation. Hence it is rewritten into a weakly singular form using a regularization technique proposed by Bonnet. The first step, following a method due to Sladek and Sladek, consists in converting the bypersingular integral equation for the displacement discontinuity into an integral equation for the displacement discontinuity and its tangential derivatives (dislocation density distribution); the latter involves a Cauchy type singular kernel. The second step is based on the observation that the hypersingularity is related to the static component of the kernel; the static singularity is then isolated and can be expressed in terms of weakly singular integrals using a result due to Bonnet. Although numerical applications discussed in this paper are all for the antiplane problem, the technique can be applied as well to in-plane crack dynamics.

The BIEM is implemented numerically using continuous linear space-time base functiolis to model the COD on the crack. in the present scheme the COD gradient interipolation is discontinuous at the element nodes while the integral equations are collocated at the element midpoints. This leads to an overdetermined discrete problem which is solved by standard leastsquares methods. We use the dynamic BIEM to study a set of problems that appear in earthquake source dynamics, including the spontaneous dynamic crack propagation for a very simple rupture criterion. The numerical results compare favorably with the few exact solutions that are available. Then we demonstrate that difficulties experienced with finite difference simulations of spontaneous crack dynamics can be removed with the use of BiEM. The results are improved by the use of singular crack tip elements.
\end{abstract}

\section{Introduction}

Many numerical studies of shear crack prupagation viewed as a possible model of shallow earthquakes have been published in the past twenty years. In this article, a new numerical technique is presented which is more generally applicable and of higher precision than those used up to now. The present numerical approach is based on a regularized time-domain traction boundary integral equation (RIE). Our objective 
is to demonstrate the potential of this method by treating the antiplane case which is simplest, both from a geometrical and a fracture mechanical point of view. We consider an initially straight two-dimensional antiplane (mode III) shear crack, embedded in an infinite linearly elastic homogeneous medium, its spontaneous propagation being governed by a simple critical stress intensity criterion. The present numerical model allows out-of-plane as well as in-plane propagation. Here the onset of a potential kinking of the crack path is studied. A focus of growing interest in seismology is the dynamic interaction between faults, both on a regional (earthquakes "jumping" from one fault segment to another, e.g. [11]) as well as on a micro scale ("en echelon" fractures). Several works have been published on static fault interaction, see e.g. $[37,29]$, but to our knowledge the dynamical interaction problem has been considered only in paper [26]. Further problems of interest that may be studied with our numerical scheme are the modelling of rupture along an existing kinked fault (e.g. El Asnam [47]) or the determination of the terminal velocity of rupture propagation on a new or considerably recrystallized fault by allowing the crack to zigzag around its overall path in analogy to what is observed in experiments with tensile cracks. Two different numerical approaches have been used so far in the quantitative study of dynamical earthquake source mechanics: a displacement BIE formulation and several finite difference (FD) techniques. Early works were those of Burridge [14], Das and Aki [17], Andrews [3] on BIE, Andrews [4], Madariaga [34], Virieux and Madariaga [44] on FD. Although both approaches were eventually applied to rather sophisticated problems, including inhomogeneous material resistance or stress fieids and three-dimensional elasticity problems associated with bounded plane cracks (see [18] for further references), all studies have dealt only with single plane cracks. It is indeed virtually impossible to treat geometrically non-planar crack problems with the displacement BIE. FD techniques, on the other hand, are not limited in this respect, although the possibilities of dealing with kinked cracks are restricted by the actual configuration of the computational grid. In fact, as pointed out in [44], the accuracy of second-order FD techniques is unsatisfactory for the study of spontaneous rupture. The main numerical problems arising concern the stress resolution near the crack tip and the numerical dispersion by the computational grid. Even the method proposed by Trifu and Radulian [43], which considerably improves the stress resolution, does not seem to erhance significantly the efficiency of FD techniques. In contrast, our regularized time-domain traction BIE, or related approaches like that of Hirose and Achenbach [27], are expected to overcome these shortcomings.

\section{Time-domain boundary integral equations for crack analysis}

Throughout the present paper, the discussion is restricted to $2 \mathrm{D}$ antiplane time-domain elasto-dynamics (shear modulus $\mu$, specific mass $\rho$ ). Zero body forces and homogeneous initial $(t=0)$ conditions are assumed. Accordingly, a domain $\Omega \subset \mathbb{R}^{2}$ of boundary $\partial \Omega$ is considered. The coordinate directions $\left(e_{1}, e_{2}, e_{3}\right)$ are chosen so that the only nonzero displacement and stress components are $u_{3}(y, \tau), \sigma_{3 \gamma}(y, \tau)(\gamma=1,2)$ respectively (in the sequel, Greek indices always take the values 1,2). The unit normal vector $n=n_{\gamma} e_{\gamma}$ is directed towards the exterior of $\Omega$. Hooke's law reduces to

$$
\sigma_{3 \gamma}=\sigma_{\gamma 3}=\mu \frac{\partial u_{3}}{\partial y_{\gamma}}(y, t), \quad \sigma_{i i}=0 \text { otherwise. }
$$

\subsection{BIE for antiplane elastodynamic crack problems}

Under these assumptions, the displacement $u_{3}(z, t)$ at an interior point $z=z_{\gamma} e_{\gamma} \in \Omega$ can be expressed in terms of boundary displacement $u_{3}$ and traction $T_{3}=\sigma_{3 \gamma} n_{\gamma}$ [22] using the following well-known integral 
representation formula:

$$
u_{3}(z, t)=-\int_{\partial \Omega} \int_{0}^{t}\left\{u_{3}(y, \tau) n_{\gamma}(y) \Sigma_{3 \gamma ; 3}(z, y, t-\tau)-T_{3}(y, \tau) U_{3: 3}(z, y, t-\tau)\right\} \mathrm{d} \tau \mathrm{d} s_{y}, \quad z \notin \partial \Omega
$$

where $U_{3 ; 3}(z, y, t-\tau)$ denotes the only nonzero component of the impulsive antiplane Green's tensor for the infinite space [22], i.e. the antiplane displacement at $y \in \mathbb{R}^{2}$ and time $t$ created by an impulsive antiplane point load applied at $z \in \mathbb{R}^{2}$ and time $\tau \leqslant t, \Sigma_{3 \gamma ; 3}(z, y, t-\tau)$ being the $(3, \gamma)$-components of the elastic stress tensor associated with $U_{3 ; 3}(z, y, t-\tau)$ through Hooke's ia $\because$ (1). The analytical expressions of antiplane Green's tensors are given by eqs. (47) and (48) oi Appendix A. In the sequel, the field components $u_{3}, T_{3}$, $U_{3 ; 3}, \Sigma_{3 \gamma ; 3}$ will be simply denoted by $u, T, U, \Sigma_{\gamma}$, the unnecessary '3' indices being dropped for convenience.

The representation formula (2) is the basis for boundary integral equation (BIE) methods for $2 D$ antiplane nroblems. Among them, the well-known and widely used displacement BIE $[7,12]$ is obtained through a limiting process $z \rightarrow x \in \partial \Omega$, where the internal source point $z$ is moved towards a fixed point $x$ located on the boundary in (2) (see e.g. $[7,12,23]$ ) (the distinct notations $x, z$ will be used as a reminde of this limit process). The integrations involving the kernels $\Sigma_{\gamma}(z, y, t-\tau)$ are then to be understood as Cauchy principal values (CPV). The actual numerical evaluation of CPV integrals has been a source of numerical difficulties, which were overcome recently using either regularization approaches $[8,36]$ or a direct numerical treatment of general CPV integrals [24].

However the BIE modelling of crack problems, in the framework of elastic fracture theory, involves further specific difficulties, which are not present when considering non-cracked domains. Let from now on $\Omega$ be an unbounded 2D elastic domain containing a crack (upper and lower faces $C^{+}$and $C^{-}$respectively): $\partial \Omega=C^{+} \cup C^{-}, C^{+}$and $C^{-}$being in fact the sanie geometrical curve $C=(A B)$ in the $x_{1} x_{2}$-plane with opposite unit normals $n^{+}, n^{-}$(Fig. 1). The unit normal vector $n^{-n^{-}}=\left(n_{1}, n_{2}\right)$ will be used, together with the unit tangent vector $\tau=\left(\tau_{1}, \tau_{2}\right)$ defined by

$$
\tau_{\gamma}(y)=n_{\beta}(y) e_{\gamma \beta}
$$

where $e_{\gamma \beta}$ denotes the two-dimensional permutation symbol. The curvilinear abscissa along $C$ is denoted by $s$, with $s=0$ for the endpoint $A$. The faces of the crack are loaded by tractions $T^{+}, T^{-}$(the crack faces are physically considered free of tractions, but one often puts $T^{ \pm}=-\sigma_{3 \gamma}^{\text {ref }} n_{\gamma}$ in order to consider the perturbation induced by the presence of the crack on a reference elastic state $u^{\text {ref }}, \sigma^{\text {ref }}$ known everywhere in $\Omega$ ).

Let us introduce the notation $\phi(y, \tau)=u\left(y^{+}, \tau\right)-u\left(y^{-}, \tau\right)$ for the crack opening displacement (COD); in the subsequent discussions, $\phi$ will be also sometimes termed as "slip", as is usually done in geophysics. Taking into account $\partial \Omega=C^{+} \cup C^{-}, n^{+}=-n^{-}=-n, T^{+}=-T^{-}$, the representation formula (2) leads to

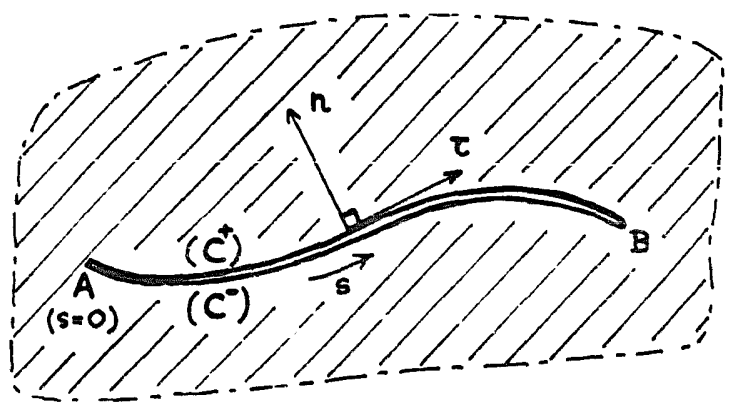

Fig. 1. Two-dimensional crack problem: geometry and notations. 
the following integral representations of the displacement and (upon application of Hooke's Law (1)) stresses for the antiplane crack problem:

$$
\begin{aligned}
& u(z, t)=\int_{C} \int_{0}^{t} \phi(y, \tau) n_{\gamma}(y) \Sigma_{\gamma}(z, y, t-\tau) \mathrm{d} \tau \mathrm{d} s_{y}, \quad z \notin C \\
& \sigma_{3 \beta}(z, t)=-\mu \int_{C} \int_{0}^{t} \phi(y, \tau) n_{\gamma}(y) \frac{\partial}{\partial y_{\beta}} \Sigma_{\gamma}(z, y, t-\tau) \mathrm{d} \tau \mathrm{d} s_{y}, \quad z \notin C .
\end{aligned}
$$

In view of the well-known degeneracy of the displacement BIE, which is due to the fact that the boundary data $T^{+}, T^{-}$do not appear explicitly in (4), an alternative possibility is the statement of a iraction BIE, in order to relat explicitly the traction data to the $\operatorname{COD}[8,9,13,27,35,38,46,48,49]$. The latter is obtained by a limiting process $(z \notin C) \rightarrow(x \in C)$ in (5). Due to the hypersingularity of the kernel components $\left(c_{/} / \partial_{y_{\beta}}\right) \Sigma_{\gamma}(\tau, y, z \cdots \tau)$ for $:=\tau$ and $z=y$, the resulting hypersingular integral over $C$ is understood as a finite part (FP).

Because of the hypersingularity problem, which is a considerable obstacle to the numerical implementation of the traction BIE, researchers formerly developed and applied alternative approaches to dynamical crack analysis, leading to BIE involving integrable singularities, but having less generality regarding the geometry of the problems under consideration. Many of these works were motivated by the study of seismological problems. For example, when considering planar cracks in infinite elastic domains, the problem can be stated, using symmetry with respect to the crack plane $P$, on the half-space $\Omega^{+}$bounded by $P$ (this is a particular case of "substructuring"). One can then apply the displacenient 2 !E to the domain $\Omega^{+}$, using the half-space Green's tensor satisfying a free-surface (zero traction) condition on $\vec{i}$. This leads to a weakly singular displacement BIE, in which the unknown is the traction $T$ on $P-C$. However, tiis method allows only the study of straight cracks, forbidding the consideration of out-of-plane propagation of kinking, originally non-straight cracks, interaction between non-collinear cracks, etc. Using this approach, Burridge [14] studicd dynamically loaded fixed cracks in antiplane arid plane strain, while Das and Aki [17], Andrews [5] considered propagating cracks, either spontaneously or at fixed velocity, the latter introducing a slip-dependent friction law on the crack plane. The Green's tensor associated to a given domain $\Omega$ is generaily now known, the half-space case being an exception, thus the kind of approach reported here is hardly generalizable. Moreover any displacement FIE using a Green's tensor associated to a domain $\Omega^{\prime}$ greater than $\Omega$ (generally $\Omega^{\prime}=\mathbb{R}^{3}$ ) contains CPV integrals.

\subsection{Regularization of traction $\mathrm{BIE}$ for $2 \mathrm{D}$ antiplane crack problems}

The application of collocation traction BIE to the numerical study of crack problems requires careful handling of the hypersingula integrals. One approach consists in a direct use of the FP traction BIE obtained from (5) together with a suitable treatment of the elementary FP integrals [25] in which the mapping between physical element and reference element carefully preserves the symmetrical vanishing exclusion ne:ghborhood used to define the value of FP integrals. Another approach consists in rewriting the hypersingular BIE, using a regularization approach, into an equivalent form which involves only weakly singular integrals. The regularization of general three-dimensional elastodynamic traction BIE is treated in $[9,10]$, see also [32]. Let us finally mention for completeness that regularization can also be achieved by deriving a Galerkin-type variational BIE, see e.g. [6] for time-domain crack problems.

The present work is an application of the regularization of collocation traction BIE to the numerical simulation of 2D propagating cracks under antiplane shear. The regularization process [9] leading to a weakly singular traction BIE is outlined as follows. 
- The firs: step is due to Sladek \& Sladek [38]. The inner product of the representation formula (5) of $\sigma_{3 \gamma}(z, t)$ with $n^{ \pm}(x)$ (for fixed $z \notin C$ and $x \in C$ ) is t: ken. Then the resulting equation is integrated by parts with respect to $y$ (no singularity occurs at this stage). Finally, in order to relate the given tractions $T^{ \pm}$ on $C$ to the unknown COD $\phi$, the limiting process $(z \notin C) \rightarrow(x \in C)$ is carried out, giving as a result

$$
\begin{aligned}
T^{ \pm}(x, t)=-\mu n_{\beta}^{ \pm} & \left\{\int_{C}^{*} \int_{0}^{t} \Sigma_{\gamma}(x, y, t-\tau) e_{\gamma \beta} \frac{\partial}{\partial s} \phi(y, \tau) \mathrm{d} \tau \mathrm{d} s_{y}\right. \\
& \left.+\rho \int_{C} \int_{0}^{t} U(x, y, t-\tau) \ddot{\phi}(y, \tau) n_{\beta}(y) \mathrm{d} \tau \mathrm{d} s_{y}\right\} .
\end{aligned}
$$

In eq. (6) and the sequel, the asterisk (*) denotes a CPV integral. The CPV traction BIE (6) generalizes, for the two-dimensional case, the results of Bui [13], Weaver [46] in elastostatics. Zhang and Achenbach [49], Zhang [48] also obtained the BIE (6) for two- or three-dimensional problems using the concept of $\tilde{J}_{k}$ conservation integral.

- Then the CPV traction BIE (6) is regularized as follows. First eq. (6) is rewritten into the following equivalent equation $[8,9]$ :

$$
\begin{aligned}
T^{ \pm}(x, t)= & -\mu n_{\beta}^{ \pm}(x)\left\{\int_{C}\left(\int_{0}^{t} \Sigma_{\gamma}(x, y, t-\tau) e_{\gamma \beta} \frac{\partial}{\partial s} \phi(y, \tau) \mathrm{d} \tau-\Sigma_{\gamma}^{0}(x, y) e_{\gamma \beta} \frac{\partial}{\partial s} \phi(y, t)\right) \mathrm{d} s_{y}\right. \\
& +\int_{C}\left[e_{\gamma \beta} \frac{\partial}{\partial s} \phi(y, t)-e_{\gamma \beta} \frac{\partial}{\partial s} \phi(x, t)\right] \Sigma_{\gamma}^{0}(x, y) \mathrm{d} s_{y} \\
& \left.+\rho \int_{C} \int_{0}^{t} n_{\beta}(y) U(x, y, t-\tau) \ddot{\phi}(y, \tau) \mathrm{d} \tau \mathrm{d} s_{y}+e_{\gamma \beta} \frac{\partial}{\partial s} \phi(x, t) \int_{C}^{*} \Sigma_{\gamma}^{0}(x, y) \mathrm{d} s_{y}\right\}
\end{aligned}
$$

in which $\Sigma_{\gamma}^{0}(x, y)$ denotes the static counterpart of $\Sigma_{\gamma}(x, y, t-\tau)$ (given by eq. (49) in Appendix A), i.e. the stress kernel associated to an antiplane static unit point ferce. All integrals but the last in (7) are regular or weakly singular; this relies crucially upon the equality of the singular parts, for $y=x$, of the dynamic and static kernels $\Sigma(x, y, t-\tau)$ and $\Sigma^{0}(x, y)$, and also on the assumption that $\left(\partial / \partial_{s}\right) \phi(y, t)$ is $C^{0, \alpha}, \alpha>0$, i.e. Hölder-continuous with positive exponent, on $C[32,10]$. The residual $\mathrm{CPV}$ integral in (7) can be rewritten $[8,9]$, by means of an analytic transformation using integration by parts, into a weakly singular form; the result is

$$
\int_{C}^{*} \Sigma_{\gamma}^{0}(x, y) \mathrm{d} s_{y}=-\frac{1}{2 \pi}\left\{\left[\tau_{\gamma} \log r\right]_{A}^{B}+\int_{C}\left[\frac{1}{r} \frac{\partial r}{\partial r}-\frac{1}{R(y)} \log r\right] n_{\gamma}(y) \mathrm{d} s_{y}\right\}
$$

where $r=\|y-x\|=\left(y_{\alpha}-x_{\alpha}\right)\left(y_{\alpha}-x_{\alpha}\right)$ and $R(y)$ denotes the radius of curvature at $y$. The integrand of (8), inchuding the term $(1 / r)\left(\partial_{r} / \partial_{n}\right)$, is integrable, assuming $C$ to be twice continuously differentiable (this assumption is necessary since (8) involves the curvature $R$ ). The line integral over $C$ in (8) vanishes if $C$ is a straight element. Equations (7) and (8) define the regularized traction BIE for two-dimensional antiplane crack problems. It is equivalent to the hypersingular traction BIE obtained from (5) but contains only integrable (in the ordinary sense) singularities, thus being suited for applications. This result has full generality and applies to cracks of arbitrary (sufficiently regular) shape subjected to arbitrary loadings. Indeed it can be shown [9] that equations (7) and (8) are valid, without modification, 
for the more general situation of piecewise regular (i.e. kinked, branched or crossed) cracks; in this case, each regular component of $C$ has to be twice continuously differentiable.

In practice, it is convenient to divide the curve $C$ into two complementary parts $\Delta C(x)$ and $C-\Delta C(x)$, where $\Delta C(x)$ is any subset of $C$ containing $x$ as an interior point, in order to perform the singularity cancellations and evaluate the residual integral (8) on a curve smaller than $C$ (e.g. the single boundary element containing the singular point $\boldsymbol{x}$ ). This leads to the following developed expression of the regularized traction PIr. obtained through insertion of (49), (51), (50), (3) and (8) in (7):

$$
\begin{aligned}
& \left.r^{ \pm}, \boldsymbol{x}, t\right)=\frac{\mu}{2 \pi} \tau_{\beta}^{ \pm}(\boldsymbol{x}) \\
& \quad \times\left\{\int_{\Delta C(x)} \frac{y_{\beta}-x_{\beta}}{c^{2}}\left[\frac{\partial \phi}{\partial s}(y, t-r / c)-\frac{\partial \phi}{\partial s}(y, t)\right] \mathrm{d} s_{y}+\int_{\Delta C(x)} \frac{y_{\beta}-x_{\beta}}{r^{2}}\left[\frac{\partial \phi}{\partial s}(y, t)-\frac{\partial \phi}{\partial s}(x, t)\right] \mathrm{d} s_{y}\right. \\
& \quad-\frac{i}{c^{2}} \tau_{\beta}(y) \int_{C} \int_{r / c}^{t} \ddot{\phi}(y, t-\tau) \frac{\mathrm{d} \tau}{\left(\tau^{2}-r^{2} / c^{2}\right)^{1 / 2}} \mathrm{~d} s_{y} \\
& +\frac{\partial}{\partial s} \phi(x, t)\left\{\left[\tau_{\beta} \log r\right]_{A}^{B}+\int_{C}\left[\frac{1}{r} \frac{\partial r}{\partial n}-\frac{1}{R} \log r\right] n_{\beta}(y) \mathrm{d} s_{y}\right\} \\
& +\int_{C-\Delta C(x)} \frac{y_{\beta}-x_{\beta}}{r^{2}} \frac{\partial \phi}{\partial s}(y, t-r / c) d s_{y} \\
& \left.+\int_{C} \frac{y_{\beta}-x_{\beta}}{c^{2}}\left[\int_{r, c}^{t} \frac{\partial \ddot{\phi}}{\partial s}(y, t-\tau) \frac{\mathrm{d} \tau}{\left(\tau^{2}-r^{2} / c^{2}\right)^{1 / 2}\left[\tau+\left(\tau^{2}-r^{2} / c^{2}\right)^{1 / 2}\right]}\right] \mathrm{d} s_{y}\right\}
\end{aligned}
$$

which serves as a basis for the numerical work presented in this paper.

\section{Boundary element discretization for cracks in antiplane strain}

For expository convenience, the present section describes the discretization scheme for a steady crack. The numerical management of a propagating crack will be described in the next section.

\subsection{Boundary element discretization scheme: description and notations}

The crack curve $C$ is approximated by $J$ straight elements $E_{1}=\left[y_{0} y_{1}\right], \ldots, E_{J}=\left[y_{J-1} y_{J}\right]$ of equal length $\Delta x$, and a constant time step $\Delta t$ is used $\left(y_{0}, y_{J}\right.$ are the endpoints $\left.A, B\right)$. We denote by $s$ the arc-length along $C, s_{0}=0, s_{1}=\Delta x, \ldots, s_{J}=J \Delta x$ denoting the curvilinear abscissae of the subdivision points $y_{0}, \ldots, y_{J}$. The collocation points $x_{i}=1 / 2\left(y_{1-1}+y_{i}\right)$ (i.e. the midpoints of the elements $\left.E_{1}, \ldots, E_{J}\right)$ are introduced; their curvilinear abscissae are $s_{i-1,2}=s_{i-1}+\Delta x / 2$. The regularized traction BIE (9) is collocated at the points $x_{i}$ and the time steps $t=t_{1}=\Delta t, \ldots, t=t_{N}=N \Delta t, \Delta C(x)$ being chosen as the boundary element $E_{i}$ containing the current collocation point $x_{i}$, while the COD $\phi$ is discretized in the usual manner:

$$
\phi(y, t)=\sum_{j=1}^{j-1} \sum_{m=1}^{N} v^{j}(s) \theta_{m}(t) \phi^{m} .
$$


The functions $v^{j}$ and $\theta_{m}$ are chosen piecewise linear and satisfy

$$
v^{j}\left(s_{k}\right)=\delta_{j k}, \quad \hat{\theta}_{m}\left(t_{n}\right)=\delta_{m n}
$$

so that the $v^{j}$ and $\theta_{m}$ can be written using a single basis function $S$ defined on $[-1,1]$ :

$$
v^{j}(s)=S\left(\frac{s-s_{j}}{\Delta x}\right)=S\left(\frac{s}{\Delta x}-j\right), \quad \theta_{m}(t)=S\left(\frac{t-t_{m}}{\Delta t}\right)=S\left(\frac{t}{\Delta t}-m\right)
$$

with

$$
S(u)= \begin{cases}1+u, & -1 \leqslant u \leqslant 0 \\ 1-u, & 0 \leqslant u \leqslant 1\end{cases}
$$

In order to incorporate the square-root spatial variation of the COD near the end-points of the crack, however, the functions $v^{1}(s)$ and $v^{J-1}(s)$ are modified as follows:

$$
\begin{aligned}
& v^{1}(s)= \begin{cases}{[s / \Delta x]^{1 / 2},} & 0 \leqslant s \leqslant \Delta x \\
S(s / \Delta x-1), & \Delta x \leqslant s \leqslant 2 \Delta x\end{cases} \\
& v^{J-1}(s)= \begin{cases}S(s / \Delta x-(J-1)), & (J-2) \Delta x \leqslant s \leqslant(J-1) \Delta x \\
{[J-s / \Delta x]^{1 / 2},} & (J-1) \Delta x \leqslant s \leqslant J \Delta x .\end{cases}
\end{aligned}
$$

The present discretization scheme yields $J$ equations for $J-1$ scalar unknowns. This is due to the fact that collocation at element endpoints is impossible using the present choice (12) of shape functions $v^{j}$ because, as has been mentioned above, the CPV integral eq. (6) as well as its regularized counterpart (9) are valid only for collocation points $\boldsymbol{x}$ at which $(\partial / \partial s) \phi(y, t)$ is $C^{0, \alpha}$. This restriction extends to $C^{0}$ polynomial interpolation of any degree, the number of degrees of freedom and of equations matching only for piecewise constant spatial interpolation.

The ratio $c \Delta t / \Delta x$ has been chosen equal to $1 / 2$. This value is smaller than $1 / \sqrt{2}$ and therefore lies within the stability range of corresponding two-dimensional FD methods [44]. Furthermore, this choice allows one to perform analytically many of the elementary integrations, especially for the singular terms or for straight parts of the crack. In another BEivi investigation of transient crack problem [35], the authors chose $c \Delta t / \Delta x=1$ and reported good results.

For a given length $L$ of the crack, the insertion of (10) into (9), with $t=t_{n}=n \Delta t$ and $x=x_{i}$, leads to the following system of equations:

$$
\frac{2 \pi}{\mu} T_{i}^{n}+\sum_{j=1}^{J-1}\left[H_{i j}^{n, n} \phi_{j}^{n}+\sum_{m=1}^{n-1} H_{i j}^{n, m} \phi_{j}^{m}\right]=0, \quad n=1, \ldots, N
$$

or

$$
\frac{2 \pi}{\mu} \mathbb{T}^{n}+H^{n, n} \phi^{n}+\sum_{m=1}^{n-1} H^{n, m} \phi^{m}=0, \quad n=1, \ldots, N
$$

in matrix form. The coefficients $H_{i j}^{n, m}$ of (16) are given by

$$
H_{i j}^{n, m}=H V_{i j}^{n, m}+H G_{i j}^{n, m}+H A_{i j}^{n, m}
$$


with

$$
\begin{aligned}
& H V_{i j}^{n, m}=\tau_{\beta}(x) \frac{1}{c^{2}} \int_{C}\left(y_{\beta}-x_{\beta}\right) v^{j}(s) \\
& \quad \times\left[\int_{r / c}^{t} \frac{\dot{S}(n-m-\tau)}{\left(\tau^{2}-r^{2} / c^{2}\right)^{1 / 2}\left[\tau+\left(\tau^{2}-r^{2} / c^{2}\right)^{1 / 2}\right]} \mathrm{d} \tau\right] \mathrm{d} s_{y} \\
& H G_{i j}^{n, m}=H G R_{i j}^{n, m}+H G S_{i j}^{n, m} \\
& H G R_{i j}^{n, m}=\tau_{\beta}(x) \int_{C-E_{t}} \frac{y_{\beta}-x_{\beta}}{r^{2}} v^{\prime j}(s) S(n-m-r / c \Delta t) \mathrm{d} s_{y} \\
& H G S_{i j}^{n, m}=0, \quad m<n-1 \\
& H G S_{i j}^{n, n-1}=-\tau_{\beta}(x) \int_{E_{i}} \frac{y_{\beta}-x_{\beta}}{r^{2}} v^{\prime j}(s)[S(-r / c \Delta t)-1] \mathrm{d} s_{y} \\
& H G S_{i j}^{n, n}=\tau_{\beta}(x)\left\{\int_{E_{i}} \frac{y_{\beta}-x_{\beta}}{r^{2}}\left[v_{j}^{\prime}(s)-v_{j}^{\prime}\left(s_{i-1 / 2}\right)\right] \mathrm{ds} y+\left[\tau_{\beta} \log r\right]_{s_{i}-1}^{s_{i}}\right\}-H G S_{i j}^{n, n-1} \\
& H A_{i j}^{n, m}=\tau_{\beta}(x) \frac{1}{c^{2}} \int_{C} \tau_{\beta}(y) v^{j}(s)\left[\int_{r / c}^{t} \frac{\ddot{S}(n-m-\tau)}{\left(\tau^{2}-r^{2} / c^{2}\right)^{/ 2}} \mathrm{~d} \tau\right] \mathrm{d} s_{y} .
\end{aligned}
$$

\subsection{Comments about the computation of the elementary integrals (18) to (25)}

Equations (18) to (25) deserve some comments and a closer examination.

1. Equations (23)-(24) take into account the specific value $1 / 2$ of the ratio $c \Delta t / \Delta x$ used here.

2. The substitution (12) has been made for the time basis functions $\theta_{m}$ in (19), (21), (22), (23), (24), (25), in order to make apparent that the elementary integrals $H_{i j}^{n, m}$ possess the translation property

$$
H_{i j}^{n, m}=H_{i j}^{n-m, 0}
$$

which is the discrete equivalent of the convolution structure of the time-domain BIE. $H_{i j}^{n, m}$ will be accordingly denoted by $H_{i j}^{n-m}$.

3. The process of removing the spatial singularity of the Stokes tensors appears in the elementary integrals $H G S_{i j}^{n, n}$ and $H G S_{i j}^{n,(n-1)}$ eqs. (23)-(24): the $1 / r$ singularity is cancelled out by $v_{j}^{\prime}(s)-v_{j}^{\prime}\left(s_{i-1 / 2}\right)$ or $S(-r / c \Delta t)-1$. These integrais can be evaluated analytically for the present discretization method. First, the elements are straight segments and $\boldsymbol{x}$ is located at the center of an element; as a consequence, the bracketed tern (i.e. the residual CPV integral (8)) vanishes. Then, our spatial interpolation choice for $\phi$ implies that

$$
\left[\frac{\partial}{\partial s} \phi(y, t)-\frac{\partial}{\partial s} \phi(x, t)\right]=0 \text { on } E_{i}, \quad 2 \leqslant i \leqslant J-2 .
$$


Equation (27) does not hold for $\boldsymbol{x}=\boldsymbol{x}_{1}$ and $\boldsymbol{x}=\boldsymbol{x}_{j}$ i.e. for the two end elements, because of the use of the crack-tip basis functions (14) which are not linear. Finally, the remaining integrals in (23)-(24) are evaluated analytically in Appendix B. The analytic expressions (70) are the materialization, for the present formulation, of our singularity extraction approach which led to BIEs (7) and (9); all other elementary integrals are regular. The only nonzero terms in (70) are associated to the crack-tip basis functions; this is specific of the present interpolation scheme (12) and does not extend to higher-degree basis functions or collocation at non-centered points.

4. The inner integrals with respect to time in eqs. (19) and (25) can be straightforwardly expressed in terms of elementary functions, irrespective of the actual shape of the crack; this results in eqs. (52) and (53) of Appendix A.

5. The outer spatial integrations over the elements $E_{i}$ in (19), (21) and (25) are to be performed. At this stage a technical difficulty arises, because the integrands, considered as functions of $s$, have discontinuous derivatives at points such that $r / c=k \Delta t$ ( $k$ integer). Therefore the standard numerical integration methods, e.g. Gaussian, are likely to give poor results if applied over the whole element $E_{i}$. Instead one has to partition each element $E_{i}$ into subelemeits $E_{i}^{k}$ :

$$
E_{i}=\bigcup E_{i}^{k} \quad \text { with } E_{i}^{k}=\left\{y \in E_{i},(k-1) \Delta t \leqslant r / c \leqslant k \Delta t\right\} .
$$

Each collocation point $\boldsymbol{x}$ is associated to a different partition (28). Given $\boldsymbol{x}$ and $E_{i}$, (28) involves obviously a finite number of subelements $E_{i}^{k}$. Then an elementary integral over $E_{i}$ is the sum of "subintegrals" over the $E_{i}^{k}$, each "subintegral" being evaluated using standard numerical methods, or analytical methods in special cases.

This technical difficulty is common to all BEM development using retarded potentials and timemarching schemes. The partition (28) is clearly a purely geometrical problem (find the intersection points of a given curve $C$ with circles centered at a given point $x$ and $f$ equally spaced radii). It is reasonably tractable for two-dimensional problems. On the contrary, its three-dimensional equivalent (find the intersection curves of a given surface $S$ with spheres centered at a given point $\boldsymbol{x}$ and of equally spaced radii) is extremely complicated and, to our best knowledge, a numerically efficient treatment of this particular point is still an open problem (see however [30], where the spatial integrations are performed by partitioning the elements into very small rectangular subelements).

6. The spatial elementary integrations in (19) to (25), on each subelement, are done using a numerical method. Integrals containing the linear basis functions $v^{j}(s)(2 \leqslant j \leqslant J-2)$ are evaluated using an ordinary Gaussian method (we took 10 points per subsegment), while a Gauss-Chebyshev method has been used for the crack-tip basis functions in order to integrate accurately the square-root singularity of the COD gradients.

In the particular case of straight cracks, the elementary integrals have for any value of $c \Delta t / \Delta x$ the following spatial translation property:

$$
H_{i j}^{n-m}=H_{i-j}^{n-n}=-H_{j+i-i}^{n-m}
$$

which stems from the uniformity of the space grid. Thus the values of the elementary integrals have to be computed for only one collocation point. The translation property (29) does not apply to integrals involving the crack-tip basis functions (i.e. for $j=1$ or $j=J-1$ ), which must therefore be evaluated for each collocation point. All the elementary integrals for a straight crack have been evaluated analytically, these calculations being less complicated when a simple value for the ratio $c \Delta t / \Delta x$ (like the present choice $1 / 2$ ) is used. 


\subsection{Time-marching scheme}

The system of equations (17) is rewritten as

$$
H^{0} \phi^{n}=-\frac{2 \pi}{\mu} T^{n}-\sum_{m=1}^{n-1} H^{n-m} \phi^{m}, \quad n=1, \ldots, N
$$

The matrix relation (30) exhibits the features classically encountered in time-marching schemes:

1. At each time step, only one new matrix has to be computed, namely $\boldsymbol{H}^{n-1}$.

2. The whole process needs the factorization of $\bar{H}^{0}$ oniy.

3. A new right-hand side has to be computed at each time step, involving all the past values of the COD. Due to the discretization scheme introduced in Section 3, the system (30) has $J$ equations for $J-1$ unknowns and is hence overdetermined. As a consequence, (30) is solved for $\phi^{n}$ in the least-squares sense, as

$$
\min _{\phi^{n}}\left\|H^{0} \phi^{n}+\frac{2 \pi}{\mu} T^{n}+\sum_{m=1}^{n-1} H^{n-m} \phi^{m}\right\| .
$$

The matrix $\boldsymbol{H}^{0}$ is factorized only once, by means of the Householder algorithm [40] and using the subroutine SQRDC of the software library LINPACK [21]. This method uses orthogonal transformations. Hence the condition number of $\boldsymbol{H}^{0}$ is preserved, while it would have been squared, and hence deteriorated, using the classical normal equations of linear least-squares.

Then (31) is solved at each time step, using the previously factorized matrix $\boldsymbol{H}^{0}$ and the LINPACK subroutine SQRSL [?1].

\section{Numerical simulation of the crack growth}

We consider here the unilateral propagation of an initially straight crack, where tip $A$ is kept fixed. Tip $B$ propagates either with a prescribed rupture velocity or spontaneously (i.e. according to a dynamical rupture criterion, similarly to [17] or [44]). The propagation is simulated by adding new elements to the crack ahead of the current location of the tip, which thus advances by discrete jumps of length $\Delta x$.

\subsection{Numerical simulation of the crack propagation}

Crack tip propagation is managed as follows. The crack and the COD are initially discretized as described in Section 3, using $J_{0}$ elements of length $\Delta x$. The propagation is simulated by adding new elements to the crack ahead of the current location of the tip, which thus advances by discrete jumps of length $\Delta x$.

Let us consider the situation where $k-1(k \geqslant 1)$ new elements have been added to the initial crack since propagation started: the current crack length is thus $J_{k-1} \Delta x=\left(J_{0}+k-1\right) \Delta x$, and the current crack configuraiton is labelled $\left(C_{k-1}\right)$. The time-marching process is continued on configuration $\left(C_{k-1}\right)$ until the time step for which the rupture criterion (to be described in Sections 4.2 and 4.3 below) is fulfilled. This time siep is labelled $n_{k}$, and a new straight element (the $k$ th) is immediately added ahead of the current location of the propagating tip. As a consequence of the linear time interpolation (13), a new square root singularity buiids up at the new crack tip while the singularity at the previous tip vanishes linearly in time, within the next time interval $\left[n_{k} \Delta t,\left(n_{k}+1\right) \Delta t\right]$. Then this new configuration $\left(C_{k}\right)$ is maintained until the next time step $n_{k+1}$ at which the rupture criterion is verified again. 


\subsection{In-plane spontaneous propagation}

For the sake of simplicity and comparison, Irwin's fracture criterion, generalized to dynamical problems [23], is used. It states that the crack tip propagates in such a manner that its stress intensity factor (SIF) $K(t)$ equals at any time $t$ a critical value $K_{\mathrm{c}}$ (called fracture toughness), which is a material constant usually assumed to be independent of rupture velocity. Close enough to the crack tip, at a distance $x_{t}$ in front of it, the stress $\sigma_{23}$ is related to $K(t)$, under very general assumptions [23], by

$$
\sigma_{23}\left(x_{t}\right)=K\left(2 \pi x_{t}\right)^{-1 / 2}+\mathrm{O}(1) \text {. }
$$

Accordingly, $K_{\mathrm{c}}$ can be approximately translated, for a fixed small $x_{t}$, into a critical stress level $\sigma_{23}\left(x_{t}\right)=$ $\sigma_{c}\left(x_{t}\right)$ which has been used for the present numerical computations.

In practice, however, the stress is usually not calculated close enough to the tip (a quantitative study of the spatial extent of the region in which the stress field is actually dominated by its singular crack-tip part has been made in [33]). Thus eq. (32) associates to the physically correct value $\sigma_{c}\left(x_{t}\right)$ an estimated value $K_{t}$ of the fracture toughness which differs from its physical value $K_{\mathrm{c}}$ by an adjustment factor $\delta: K_{\mathrm{t}}=$ $(1+\delta) K_{c}([17]$, eq. $(21))$. The adjustment term $\delta$ is necessary for comparing numerical results (obtained for the critical stress $\sigma_{c}$, related by (32) to the estimated $K_{t}$ ) to analytical solutions (expressed in terms of $K_{c}$ ). Indeed this correction was used for Figs. 4 and 5 of [44] and Figs. 5 and 6 of the present paper. This adjustment was unavoidable in [17] or [44] since only a nean value of the stress over the closest grid element in front of the crack tip was numerically available. Using the present method, it would have been possible to compute the stresses at points arbitrarily close to the crack tip using the integral representation formula (5) in our numerical scheme; however, they were actually evaluated, for practical reasons, at $x_{t}=$ $\Delta x / 2$, mid-points of (future) boundary elements.

Two ways of discretizing Irwin's criterion have been tested. The first on , :eferred to as "simple", strictiy corresponds to what was done in [17] and [44]; according to it, the next jump of the discretized crack occurs at the time $n_{k+1} \Delta t, n_{k+1}$ being the first time step such that

$$
\sigma_{23}\left(x_{t}, n_{k+1} \Delta t\right) \geqslant \sigma_{c}\left(x_{t}\right) \text {. }
$$

Although reasonable at first sight - and seemingly successful in [17] and [44] - this procedure does not give a strict discretization of Irwin's criterion as formulated above, and leads to unsatisfactory results in the present study (see Fig. 6). To see that, the time-average of $\sigma_{23}\left(x_{t}, t\right)$ since the last crack jump, denoted as $\bar{\sigma}_{23}\left(x_{t}, \tau, n_{k}\right)$, is introduced:

$$
\bar{\sigma}_{23}\left(x_{t}, \tau, n_{k}\right)=\frac{1}{\tau-n_{k} \Delta t} \int_{n_{k} \Delta t}^{\tau} \sigma_{23}\left(x_{t}, t\right) \mathrm{d} t, \text { for } \tau \geqslant n_{k} \Delta t .
$$

The "true" propagation process is such that $\tilde{\sigma}_{23}\left(x_{i}, \tau, n_{k}\right)=\sigma_{\mathrm{c}}\left(x_{t}\right)$ for every $\tau$. However. immediately after each jump occurring during the stepwise numerical simulation of the propagation, the stress $\sigma_{23}\left(x_{t}, t\right)$ in front of the new element drops to a value much smaller than $\sigma_{\mathrm{c}}\left(x_{t}\right)$; thus the time-average $\bar{\sigma}_{23}\left(x_{t}, n_{k+1} \Delta t, n_{k}\right)(34)$ at $\tau=n_{k+1} \Delta t$ is smaller than $\sigma_{c}\left(x_{t}\right)$. In this sense, the "simple" criterion does not adequately reproduce Irwin's criterion, especially for simulating the low-velocity initiation phase of the propagation. This remark remains equaily valid even in the limit $\Delta x \rightarrow 0$ if the ratio $A i / \Delta x$ is kept constant, as is usualiy done.

An improved version of the criterion, referred to as "averaged", has therefore been established. Rupture propagation is initiated after the first time step at which (33) is fulfilled. However, a further crack tip jump 
is only implemented when the time-averaged stress (34) reaches the critical value, i.e. at a time $t$ such that

$$
\bar{\sigma}_{23}\left(x_{t}, t, n_{k}\right)=\sigma_{c}\left(x_{t}\right)
$$

Let this occur during the $n$th time interval: a new crack element is introduced either after the (current) $n$th time step $\left(n_{k+1}=n\right)$ if $t>\left(n-\frac{1}{2}\right) \Delta t$, or after the (previous) $(n-1)$ th time step $\left(n_{k+1}=n-1\right)$ if $t \leqslant\left(n-\frac{1}{2}\right) \Delta t$; in the latter case the (current) $n$th time step is recomputed with the new configuration of the crack.

\subsection{Study of the onset of kinking}

For the static antiplane crack, the stress component $\sigma_{\theta 3}(\rho, \theta)$ varies like $\cos (\theta / 2)(\rho$ being kept constant, $(\rho, \theta)$ denoting polar coordinates with origin $B, \theta=0$ being the crack direction before kinking, see Fig. 2): it has a maximum in the crack direction $\theta=0$, so that a quasistatic crack under antiplane shear should propagate along its initial direction. During dynamical propagation, however, it is known [23] that the maximum of $\sigma_{\theta 3}(\rho, \theta, t)$ become flatter as $v(t)$ increases and eventually occurs for a nonzero angle $\theta$ (see Table 1); this may cause the appearance of a kink in the propagation path. Accordingly, in order to study the onset of kinking, the averaged stress $\bar{\sigma}_{\theta 3}\left(\rho, \theta, t, n_{k}\right)(34)$ is calculated for $\rho=x_{t}$ around the current crack tip $B(t)$, the rupture criterion becoming

$$
\max _{\theta} \bar{\sigma}_{\theta 3}\left(\rho, \theta, t, n_{k}\right)=\sigma_{c}\left(x_{t}\right) \text {. }
$$

If an angle $\theta_{K} \neq 0$ satisfies (36), $\theta_{K}$ is considered as a potential direction of kinking. In this case, a new crack element is added, in the direction $\theta_{\mathrm{K}}$. to the current configuration, according to the procedure developed for in-plane propagation. Further time steps are calculated for this kinked crack, until a new angle $\hat{\theta}_{K}$ satisfying criterion (36) is found again. If $\hat{\theta}_{K} \geqslant \theta_{K}$, the onset of kinking is qualified as stable and the

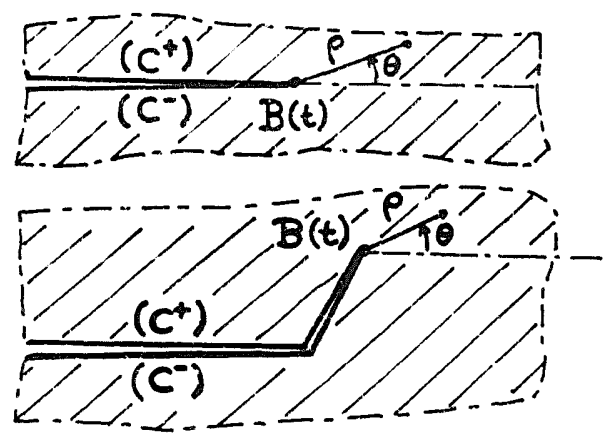

Fig. 2. Polar coordinates emanating from the propagating crack tip: (a) be' sre kinking, (b) after kinking.

Table 1

Value of the ratio $\sigma_{\theta 3}(\theta) / \sigma_{\theta 3}(0)$, against $\theta$ and $v / c$

\begin{tabular}{lllll}
\hline$v / c \theta$ & 0 & 30 & 60 & 90 \\
\hline 0.000 & 1.000 & 0.9655 & 0.8660 & 0.7071 \\
0.550 & 1.000 & 0.0970 & 0.9858 & 0.9265 \\
0.575 & 1.900 & 1.000 & 0.9997 & 0.9555 \\
0.600 & 1.000 & 1.003 & 1.015 & 0.9882 \\
0.800 & 1.000 & 1.037 & 1.207 & 1.521 \\
\hline
\end{tabular}


numerical simulation terminates; the propagation velocity observed just beforc kinking is then tentatively interpreted as a terminal velocity. If $\hat{\theta}_{\mathrm{K}} \leqslant \theta_{\mathrm{K}}$, the kinking is assumed to be unstable; the oblique element is then replaced by a straight one and the corresponding time steps are recalculated.

\section{Numerical results and discussion}

Our numerical computations and the subsequent discussions are made using the following non-dimensional quantities:

$$
\sigma^{\prime}=\frac{\sigma}{\mu}, \quad t^{\prime}=\frac{t}{\mu}, \quad K^{\prime}=\frac{K}{\mu \sqrt{\pi L}}, \quad\left(x^{\prime}, y^{\prime}\right)=\left(\frac{x}{L}, \frac{y}{L}\right), \quad \phi^{\prime}=\frac{\phi}{L}, \quad t^{\prime}=t \frac{c}{L}
$$

where $L$ is a characteristic length of the problem - either the total length of an initial finite crack or the length $\Delta x$ of a boundary element for a semi-infinite crack (where no physical characteristic length exists). For propagating cracks, $l(t)$ and $v(t)$ will respectively denote the arc-length $B(0) B(t)$ and the crack tip velocity.

Unless stated otherwise, the crack faces are assumed to be stress-free, while the only nonzero component of $\sigma^{\text {ref }}$ is $\left(\sigma_{23}^{\text {ref }}\right)^{\prime}=1$; thus the superposition method leads to consider $t_{3}^{\prime}=1$ in the BIE (9). The geophysically more realistic case of a stress drop to a constant dynamic friction level could in principle be achieved by superposing a constant stress field. However, a careful look at what happens physically if $v(t)$ becomes negative would then be mandatory.

Das and Aki [17] introduced a dimensionless quantity $S$ which normalizes the difference between the mean stress within the element which is closest to the crack tip and the homogeneous far-field stress $\sigma_{23}^{\text {ref }}$ with respect to the stress drop, in our case equal to the far field stress itself. In the case of elastic, i.e. ideally brittle crack propagation, as treated in [17, 44] and in the present paper, this quantity $S$ (called Tu in [44]) is solely an indication of how fine the numerical grid has been chosen. $S$ becomes physically significant only if a cohesive zone is introduced at the crack tip, as in [5]. In the present context, $S$ is given, using (37) and the notations of 4 , by

$$
1+S=\sqrt{2} \sigma_{c}^{\prime}\left(x_{t}=\Delta x / 2\right)=2 K_{t}^{\prime} .
$$

\subsection{Stabilization of the solution}

The first attempts to use our numerical method produced oscillations of the numerically computed COD with respect to the space and time variables. The amplitude of these oscillations increases along with time. Figure 3 (slip history of an instantaneously appearing stationary crack, modelled with 20 boundary elements) shows an example of such oscillations, where the spatial frequency is the highest allowed by the mesh (note: in all figures, the slip on the crack-tip elements shows a seemingly linear variation, but the actual computations use indeed the square-root variation). This phenomenon gets worse as more boundary elements are used.

At first sight this phenomenon could be thought of as a manifestation of an instability of the timemarching scheme, i.e. with respect to the time variable. However, the fact that the numerical COD oscillates with respect to the space variable rather suggests a spatial instability. This has been confirmed by implementing a static version of the traction BIE using the same space discretization: the static COD compuleci with the static BEM program showed the same kind of oscillations. 


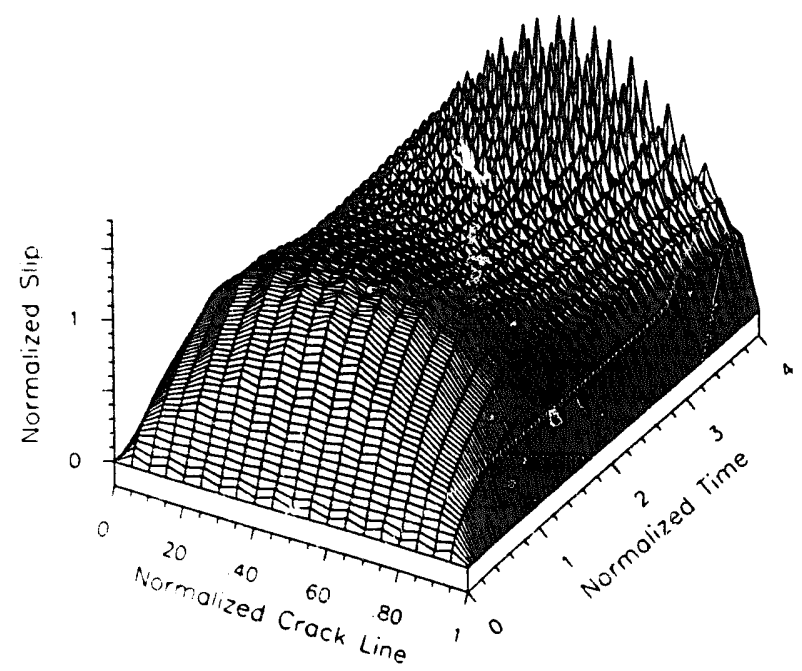

Fig. 3. Numerical solution without smoothing for an instantaneously appearing stationary crack (20 boundary elements).

This phenomenon can be explained by looking closely at the well-known static CPV traction BIE for a straight crack, which can be written using the Hilbert transform $\mathscr{H}$ (see Sneddon, [39], p. 233):

$$
\left(\forall s_{x}\right) \quad t_{3}^{ \pm}\left(s_{x}\right)+\frac{\mu}{2} \mathscr{H}\left[\frac{\mathrm{d} \phi}{\mathrm{d} s}(s) \chi(C), s_{x}\right]=0 \quad \text { with } \mathscr{H}\left[f(s), s_{x}\right]=\frac{1}{\pi} \int_{\mathscr{H}}^{*} f(s) \frac{\mathrm{d} s_{y}}{s-s_{x}}
$$

where $\chi(C)$ denotes the characteristic function of the set $C \subset \mathbb{R}$. The Hilbert transform $\mathscr{H}$ being an isometry from $L^{2}(\mathbb{R})$ to $L^{2}(\mathbb{P})([39]$, pp. 234-235), the present oscillatory phenomenon is not the kind of ill-posedness which usually affects first-kind integral equations. It is actualiy related to the fact that we collocate integral equations (9) or (39) at discrete points. It can be shown analytically (with $C=[-1,1]$ ) that

$$
\mathscr{H}\left[\sin \alpha s, s_{x}\right]=\cos \alpha s_{x}+o(1 / \alpha), \quad \mathscr{H}\left[\cos \alpha s, s_{x}\right]=\sin \alpha s_{x}+o(1 / \alpha), \quad \alpha \gg 1 .
$$

Thus, as the $J$ collocation points (for $C=[-1,1]$ and $\Delta x=2 / J$ ) are $s_{x}=s_{i-1 / 2}=(2 i-J-1) / J, i=1, \ldots, J$ in our approach, one has, taking $\alpha=J k \pi$ ( $k$ integer) in (40):

$$
\mathscr{H}\left[\cos J k \pi s, s_{i-1 / 2}\right]=\mathrm{o}(1 / J k \pi) .
$$

In other words, the perturbation $A \cos J k \pi s$ added to $\phi^{\prime}(s)$ produces almost zero tractions at the collocation abscissae $s_{i-1 / 2}$, and is therefore likely to pollute the numerical solution of (9) or (39). Furthermore, in view of (41), a finer mesh leads to increased pollution, consistently with what was observed. Such behavior is apparent in Fig. 3 (with $k=1$ ). Had the element nodes been taken as collocation points (1.e. $s_{x}=s_{i}=$ $(2 i-J) / J, i=1, \ldots, J-1)$, eq. (40) would give, with $\alpha=J k \pi / 2$,

$$
\mathscr{H}\left[\sin \frac{1}{2} J k \pi s, s_{i}\right]=\mathrm{o}(2 / J k \pi)
$$

and hence lead to the same kind of instability.

The suggests that the static traction BIE, collocated at regularly spaced points, ieads to spatial oscillations of the numerical COD, and hence that the observed oscillations are of a spatial rature. 
The initial problem of numerically solving the BIE (9) has then been reformulated as a penalized least squares problem, using the stabilizing functional (in the sense of Tikhonov [42]):

$$
2 \Omega\left(\phi, i_{n}\right)=\alpha_{1} L^{3} \int_{C}\left|\frac{\partial^{2}}{\partial s^{2}} \phi^{\prime}\left(s, t_{n}\right)\right|^{2} \mathrm{~d} s_{y}+\alpha_{2} \frac{L}{c^{2}} \int_{C}\left|\frac{\partial}{\partial t} \phi^{\prime}\left(s, t_{n}\right)\right|^{2} \mathrm{~d} s_{y}
$$

in which the scaling (37) has been used. The functional $\Omega(\phi, t)(43)$ allows smoothing with respect to space (using $\alpha_{1} \neq 0$ ) or time (using $\alpha_{2} \neq 0$ ) in order to numerically test both possibilities. Its discretized expression is

$$
\begin{aligned}
2 \Omega\left(\phi, t_{n}\right) & =\sum_{j=1}^{J-1}\left[\alpha_{1} \frac{L^{3}}{\Delta x^{3}}\left[\phi_{j+1}^{\prime n}-2 \phi_{j}^{\prime n}+\phi_{j-1}^{\prime n}\right]^{2}+\alpha_{2} \frac{L \Delta x}{c^{2} \Delta t^{2}}\left[\phi_{j}^{\prime n}-\phi_{j}^{\prime n-1}\right]^{2}\right] \\
& =\alpha_{1} \frac{L^{3}}{\Delta x^{3}}\left(W_{S} \cdot \phi^{\prime n}\right)^{t}\left(W_{S} \cdot \phi^{\prime n}\right)+\alpha_{2} \frac{L \Delta x}{c^{2} \Delta t^{2}}\left(\phi^{\prime n}-\phi^{\prime n-1}\right)^{t}\left(\phi^{\prime n}-\phi^{\prime n-1}\right)
\end{aligned}
$$

and the discrete least squares problem (44) becomes

$$
\min _{\phi^{\prime n}}\left\|\left[\begin{array}{c}
\boldsymbol{H}^{0} \\
\beta_{1} W_{S}+\beta_{2} I
\end{array}\right] \cdot \phi^{\prime n}-\left[\begin{array}{c}
b^{n} \\
\beta_{2} \phi^{\prime n-1}
\end{array}\right]\right\|
$$

with

$$
b^{n}=-\frac{2 \pi}{\mu} t^{n}-\sum_{m=1}^{N-1} H^{n-m} \cdot \phi^{m}, \quad \beta_{1}^{2}=\alpha_{1} \frac{L^{3}}{\Delta x^{3}}, \quad \beta_{2}^{2}=\alpha_{2} \frac{L \Delta x}{c^{2} \Delta t^{2}} .
$$

Numerical tests using (45) with various values of the non-dimensional coefficients $\alpha_{1}, \alpha_{2}$ have been performed. The results show that the oscillations of the numerical COD disappear by taking $\alpha_{1}>0, \alpha_{2}=0$ but persist if $\alpha_{1}=0, \alpha_{2}>0$ is used instead. This is a confirmation of the above analysis.

In all the forthcoming numerical results, the penalty method has been used with $a_{1}=0.5 \Delta x^{3} / L^{3}=$ $0 . S /\left(v_{0}\right)^{3}, \alpha_{2}=0 ; \alpha_{1}$ is a regularization parameter in the sense of Tikhonov, smali compared to unity.

\subsection{Comparisons with analytical results}

\subsubsection{Stationary crack}

A stationary crack of length $L$ appears suddenly at $t=0$. This problem has an exact solution $u(y, t)$, $\sigma(y, t)$ for $t \leqslant t_{f}(y), t_{f}(y)$ being the time at which the information of the finiteness of the crack reaches $y$ [33] $\left(t_{f}^{\prime}(y)=1\right.$ at the edges); and then again in the static limit [4i]. Figure 4 shows the numerical results for the stress at near-tip points $(\rho, \theta)$ with $\rho=0.01 L$ and $\theta=0^{\circ}, 15^{\circ}, \ldots, 75^{\circ}, 90^{\circ}$, computed using 50 boundary elements; they are within $1 \%$ of the exact solution, shown by continuous lines. The slip and the factor of dynamical overshoot of the SIF (exact value: $4 / \pi$ [1]) are obtained within $1 \%$ accuracy using only 20 elements; in this case the stress at near-tip points $(\rho=0.01 L)$ is overestimated by approximately $5 \%$. As a general trend, the fewer elements, the more overestimared the stress, and to a lesser extent the slip.

\subsubsection{Semi-infinite crack moving at prescribed velocity}

A semi-infinite crack suddenly appears along the negative $x_{1}$-axis and then propagates at a kinematically imposed constant rupture velocity $v$. The exact solution to this problem, found by Kostrov [31], has become 


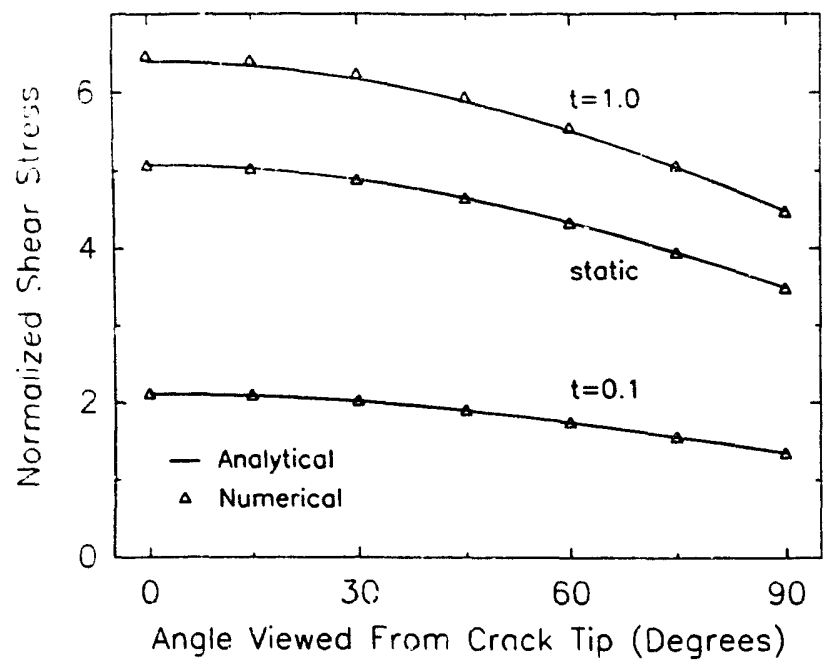

Fig. 4. Shear stress $\sigma_{\theta 3}$ for an instantaneously appearing stationary crack around its tip, at a distance of 0.01 times its total length ( 50 boundary elements).

a standard benchmark, which has been used in both [17] and [44]. Our numerical results for the displacement and stress history for a crack that instantaneously appears along $x_{1}<0$ and extends at half the shear wave velocity are compared with Kostrov's exact solution [31] and the FD results of Virieux \& Madariaga ([44], Fig. 2(a, b)) on Fig. 5. Kostrov's and our values are calculated at the points actually indicated, whereas the results of [44] are mean values ovcr one grid element. Our numerical solution agrees very well with the analytical one.

Since both slip and stress could not be computed simultaneously on the crack plane in [44], slip was evaluated for positions slightly off the plane. Its deviation from the exact solution in Fig. 5(a) might therefore be interpreted as solely due to this minor handicap. On the contrary, the stress diagram (Fig. 5 (b)) reveals a fundamental inaccuracy of the FD technique used in [44]. The seemingiy excellent stress resolution is illusory because the values shown in Fig. 5(b) are mean values over the FD grid interval closest to the crack tip. However, assuming an approximate stress distribution (eq. (28)) there, this mean
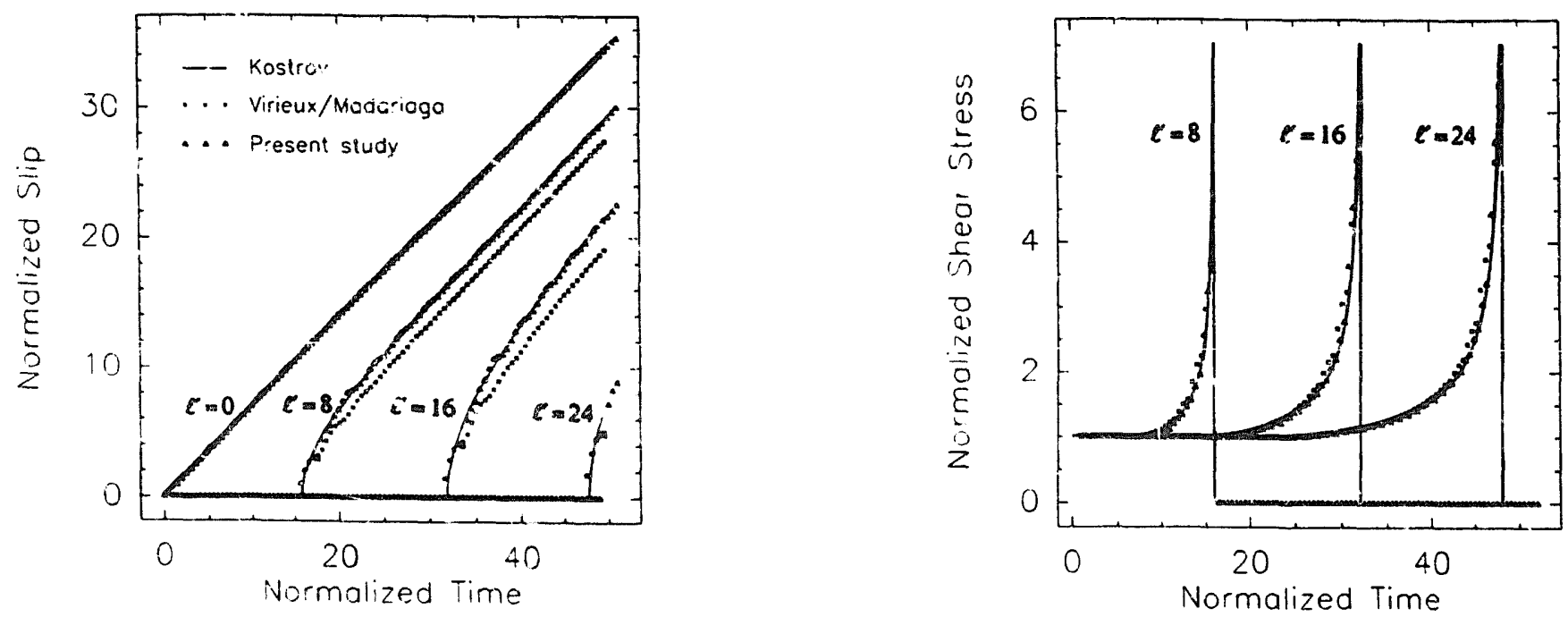

Fig. 5. Displar ment and stress history for a crack that instantaneously appears along $x_{1}<0$ and extends at $v=c / 2$. 
value should be $\sqrt{2}$ times larger than the actual stress values (computed at the grid midpoint) which are displayed for both Kostrov's and our solution. From this remark, it becomes obvious that the stress singularity is significantly better resolved by the present BIE method. It is worth noting that, owing to the self-similarity of the problem at hand, using a finer FD grid would lead to a rescaled but otherwise identical solution and could therefore not compensate for the discrepancy.

The accuracy of the slip values obtained in [17, Fig. 6] does not seem to differ significantly from the present results. However, [17, Fig. 7] shows a comparatively poorer stress resolution.

\subsubsection{Spontaneously propagating crack}

The only problem of this kind having a known analytical solution is the case of a suddenly appearing straight semi-infinite crack under antiplane shear (Kostrov [31]). The crack tip location $l(t)$ has been computed using the present BIE method; our results are displayed in Fig. 6, together with corresponding numerical results from [44], and compared to Kostrov's solution. The latter has been calculated with slightly readjusted stress intensity factors $K_{\mathrm{c}}$ of $0.50,1.85,3.93,5.90$ respectively (for the reason explained in Section 4.2), chosen to minimize the difference in slope towards the right-hand end of the parts of the curves depicted in Fig. 6. The present numerical evaluation of the early acceleration phase is excellent for $K_{\imath}^{\prime} \geqslant 2$ and if the averaged rupture criterion is used.

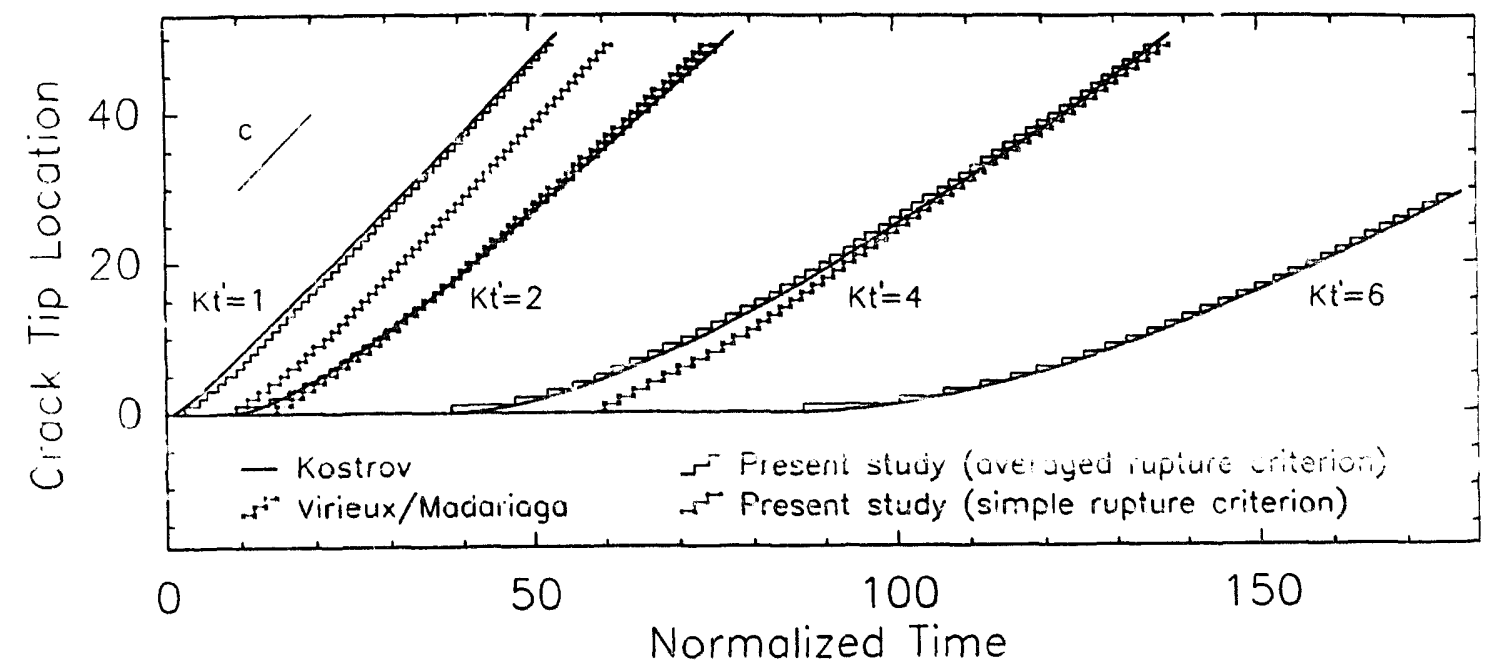

Fig. 6. Comparison of cxact [31] and numerical ([44] and present) solutions for the crack tip location during the spontaneous rupture propagation of a semi-infinite crack.

Fairly good $\left(K_{t}^{\prime}=1\right)$ to excellent $\left(K_{t}^{\prime} \geqslant 2\right)$ agreement is found when the averaged rupture criterion (see Section 4) is used. On the contrary, the simple rupture criterion makes the crack accelerate much too quickly, as it is apparent for the case $K_{t}^{\prime}=2$ in Fig. $6 . K_{t}^{\prime}=1$ should be considered as a lower bound for good results, whereas $K_{t}^{\prime}=6$ has been chosen as an upper limit for the present situation of an infinite crack: the time of fracture initiation grows with $\left(\mathbb{K}_{t}^{\prime}\right)^{2}$, and this increases not only the number of time steps but also the number of spatial elements needed to simulate effectively a semi-infinite crack (the $K_{i}^{\prime}=1$ and $K_{i}=4$ runs of Fig. 6 need respectively 2 and 15 minutes on a Cray 2 computer).

In [44], satisfactory results were obtained only for $2 \leqslant K_{t}^{\prime} \leqslant 4$; the curves for these limit values are included in Fig. 6. The poor stress resolution of the FD technique leads to a delayed fracture initiation. This delay is later compensated by a faster acceleration due to the application of the simple rupture criterion. The 
results for the same problem in [17, Fig. 13] are only evaluated for very short distances of rupture propagation. A quantitative comparison of accuracies is hardly possible using these results, particularly in view of the parameter adjustments involved. However, at least the curves for $S=4\left(K_{t}^{\prime}=2.5\right)$ and $S=5\left(K_{t}^{\prime}=3\right)$ in $[17$, Fig. 13] show clearly a tendency to accelerate too quickly, probably because of the use of the simple rupture criterion.

\subsection{Spontaneous growth of a finite crack}

Following [44], two kinds of rupture nucleation for a crack of initial length $L$ are considered here: either the crack appears suddenly at $t=0$ within a homogeneous stress field, or it is first loaded statically so that the critical stress criterion is just reached. In the latter case the crack is in metastable equilibrium, and the rupture is started by imposing a slight increase of its length, one element being added at the tip $B$ at $t=0$. This is physically more satisfactory but requires more computer time since the initial prestress field has to be calculated up to a time at which it can be considered as static.

Figure 7 shows $l(t)$ for a finite crack unilaterally extending to twice its initial length. Our results are compared with analogous ones from [44]; in both studies the initial crack was modelled with 40 elements or grid points, respectively. Although only two-sided symmetrical propagation was studied in [44], a onesided extension would have given exactly the same crack tip propagation within the time interval displayed in Fig. 7. For the instantaneous crack, Kostrov's solution for a semi-infinite crack is included, remaining valid up to $t^{\prime} \simeq 1.3$ when the information of the finite length reaches the running crack tip; after that, the numerical solutions slowly start to lag behind, as expected. In the case of the static precrack, our solution shows a very slow acceleration phase over a first short running distance; this is in complete contrast to Virieux and Madariaga's results.

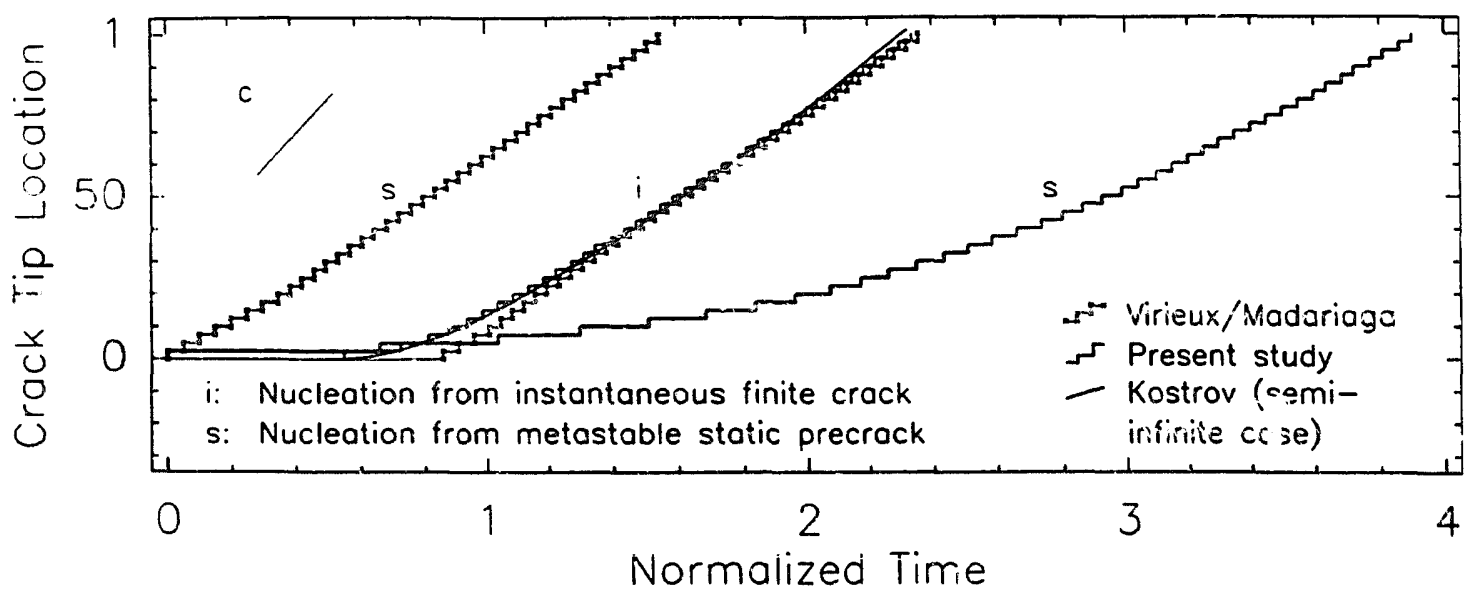

Fig. 7. One-sided spontaneous rupture from an initial finite crack ( 40 boundary elements).

The SIF of the static prestress actually obtained by the two numerical methods were respectively used as the critical value $K_{c}$. With the present normalization, this was $K_{t}^{\prime}=0.485$ in [44], 0.519 here, the corresponding analytical value being $K_{\mathrm{c}}^{\prime}=0.5$ (a small deviation in the direction of our result is expected based on the approximate caiculation of $K_{\mathrm{c}}$ from the stress as explained in Section 4 and is not a direct measure of computing precision). Renormalized for a semi-infinite crack (multiplied by $\sqrt{L / \Delta x}$ ), the analytical value would be $K_{t}^{\prime}=3.16$, i.e. within the best range for the approach of [44], see Section 5.2.

In the case of rupture propagation from an instantaneously appearing crack, both methods seem to give roughly equivalent results, although the present one resolves more accurately the early but short phase of 

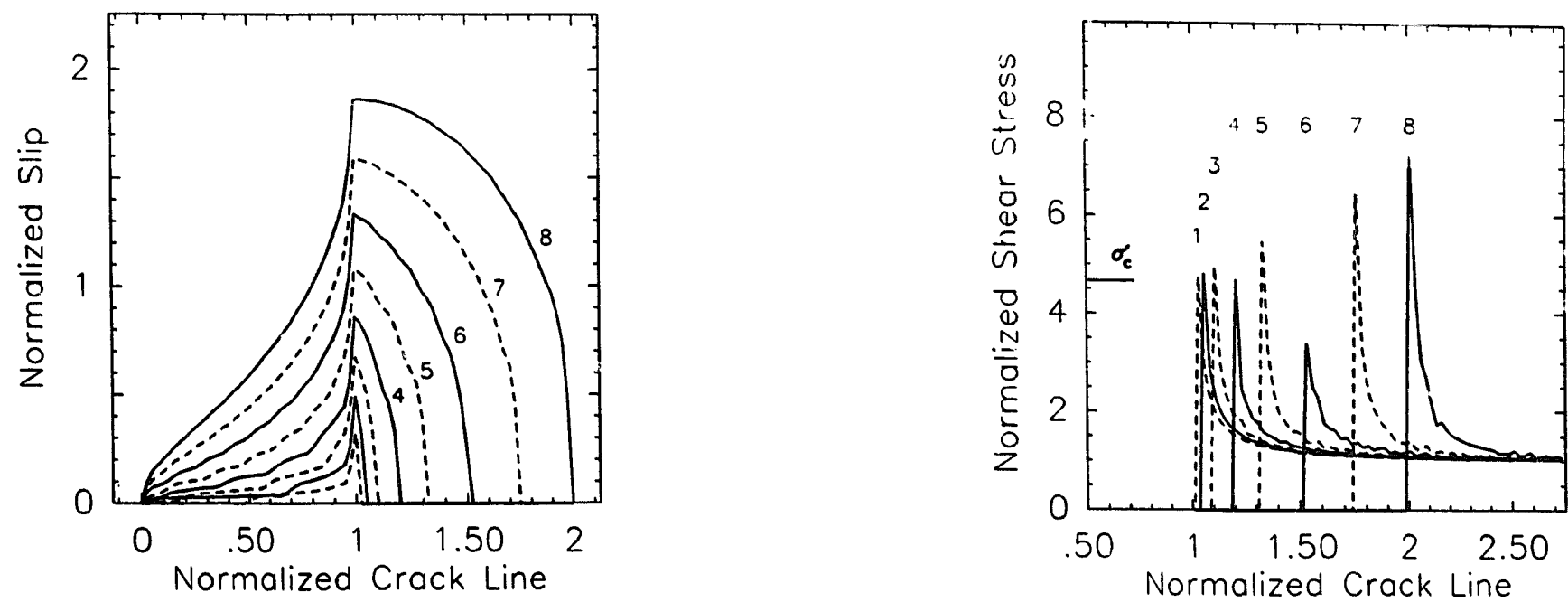

Fig. 8. Slip and stress fields for $t_{1}^{\prime}=0.5, t_{2}^{\prime}=1, \ldots, t_{8}^{\prime}=4$, for the case of our curve $s$, Fig. 7 .

noticeable acceleration. However, the situation drastically changes when looking at the physically more realistic rupture initiation from a metastable static precrack. At the time when an additional crack element is added to the static crack, the shear stress in front of the new tip is much below the critical stress $\sigma_{\mathrm{c}}$. Within only a few time steps, the stress will ultimately become larger than $\sigma_{c}$, but only slightly, since the added element is small compared to the initial crack length. It therefore takes many additional time steps until the initial stress deficiency is compensated and the fracture criterion (35) satisfied.

The slip of the crack nucleating from an initial static situation is shown in Fig. 8(a) at eight equally spaced instants $t^{\prime}=0.5,1, \ldots, 4$ (the rupture initiation taking place at $t^{\prime}=0$ ), for the crack studied in our curve $s$, Fig. 7. The crack is arrested once it has doubled its initial length. Note that the slip is measured from the initial state at $t=0$ since only this would be detectable from purely seismological measurements. The stress field $\sigma_{23}$ in front of the actual crack tip is shown in Fig. 8(b) at the same equally spaced times than in Fig. 8(a). The heights of the stress peaks vary because the times at which they are depicted are accidentally related to the instants of crack tip jumps. Nevertheless, it becomes evident that the rupture criterion (35) is satisfied by relatively low maximum stress values acting during a long time interval in early phase and by quickly growing stress acting for a shorter time once the crack moves faster.

Figure 9 shows $l(t)$ for a few examples of one-sided crack propagation in inhomogeneous media. In all cases, rupture nucleates from a metastable static precrack of length $L$ embedded in a homogeneous stress field $\left(\sigma_{23}^{\text {ref }}\right)^{\prime}=1$. The initial crack is modelled by 20 boundary elements. This turns out to be sufficient since the numerical $K_{\mathrm{c}}$ corresponds to a value $K_{f}^{\prime}=0.474$; this in turr would give a value $K_{t}^{\prime}=2.12$ in the semiinfinite case, which lies within the range of good results.

- Curve 1 corresponds to fully homogeneous conditions and therefore represents the identical physical situation as our $s$ curve in Fig. 7. The difference is that only half as many boundary elements as in Fig. 7 are used here. Since rupture is nucleated by additiou of one new boundary element in front of the static tip, the initial perturbation is now twice as big. The consequence is that the crack accelerates faster at the very beginning. However, for $l \geqslant 0.2 L$, the crack tip location histories in Figs. 7 and 9 are virtually identical, the total running times between $l=0.2 L$ and $l=L$ being equal within about $\pm 1 \%$. Thuis the results are independent of the element size, except for the effect of the element size dependent rupture nucleation which manifests itself only over a short propagation distance. 


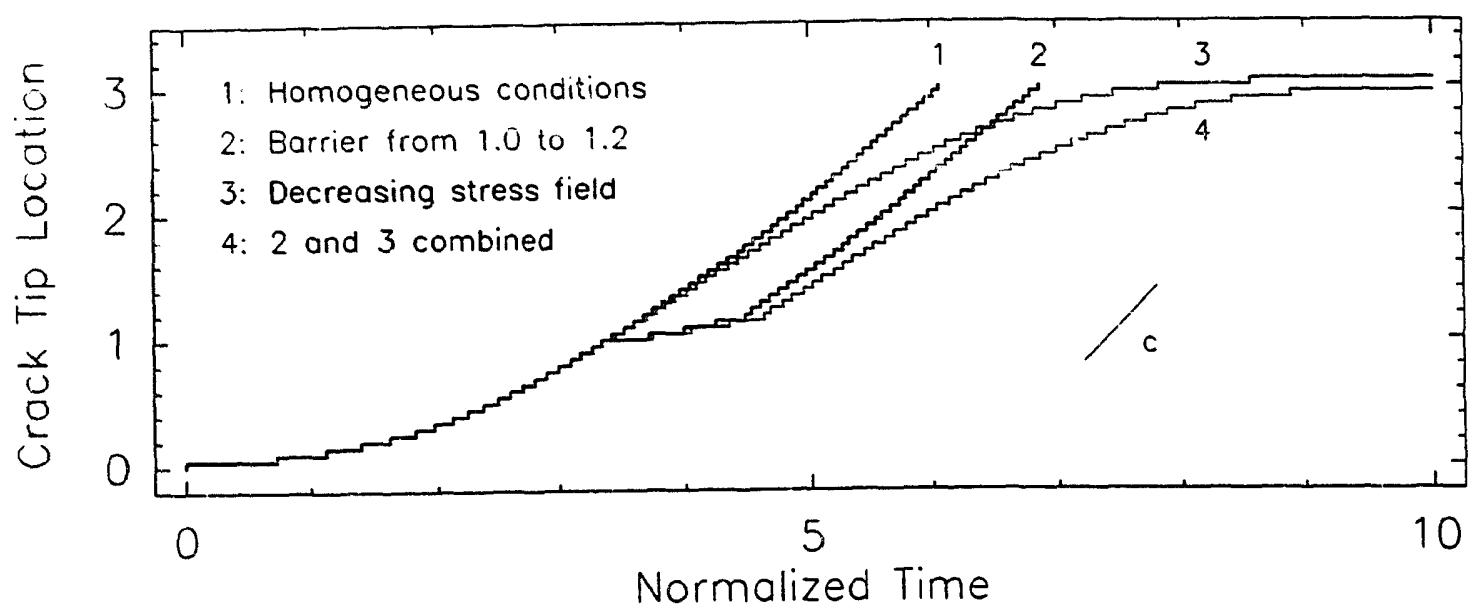

Fig. 9. Crack tip location for one-sided spontaneous rupture propagation, in different environments as indicated.

- Curve 2 represents a crack propagating within a homogeneous stress field and through a barrier of higher material resistance (the critical stress $\sigma_{\mathrm{c}}$ being $\sqrt{2}$ times larger for $L \leqslant l \leqslant 1.2 L$ than in the surrounding region), the elastic constants being unchanged. A quasistatically propagating crack reaching this barrier would find itseif in a new metastable equilibrium. The dynamical crack, however, breaks through with a low nivan velocity of less than $0.2 c$. Once ine barrier is broken, $v(t)$ seems to immediately take up the value coiresponding to the case without barrier.

- Curve 3 shows a crack running through a material of homogeneous toughness and entering a decreasing stress environment after having reached the length $2 L$, in which $\left(\sigma_{23}^{\text {ref }}\right)^{\prime}$ decreases linearly from 1.0 to 0.0 between the positions $l=L$ and $l=3 L ; v(t)$ decreases for $l \geqslant L$ and the. $\mathrm{k}$ ultimately stops at $l=3.05 L$ (its stopping near the point where the stress vanishes being due to a coincidence in the choice of the parameters).

- Curve 4 shows the combined effect of barrier and decreasing stress field. Cwing to the barrier, the crack entering the decreasing stress field loses some of its 'dynamical impetus' and ultimately stops after a slightly shorter running distance than in case 3.

The slip along the crack and the stress straight in front of it associated to curve 2 of Fig. 9 (crack breaking through a barrier) are depicted on Figs. 10(a) and 10(b) respectively. The propagation of the crack has been suddenly stopped at $l=3 L$ (equivalent to the effect of a sufficiently strong barrier). At any fixed point $x$ on the crack, the maximum value of slip occurs for the time at which the information of rupture arrest coming from the tip $B$ reaches $x$. Stresses srow remarkably immediately after the crack has stopped. Non-physical high frequency waves due to the stepwise rupture propagation can clearly be seen in both diagrams.

Figures 11(a), (b) show respectively the slip and the stress for curve 3 of Fig. 9 (decreasing ambient stress field). The crack deceleratus then stops; the stress concentration at $B$ at the end of the propagation is as strong as the initial static concentration.

Figures 10 and 11 reveal the main weakness of our numerical solution of crack growth: the stepwise propagation produces high-frequency waves, which would indeed physically exist if the real propagation process were stepwise ("pseudo-physical waves"). These waves have caused no real problem so far, but will turn out to be rather annoying when the onset of crack path kinking is studied. 

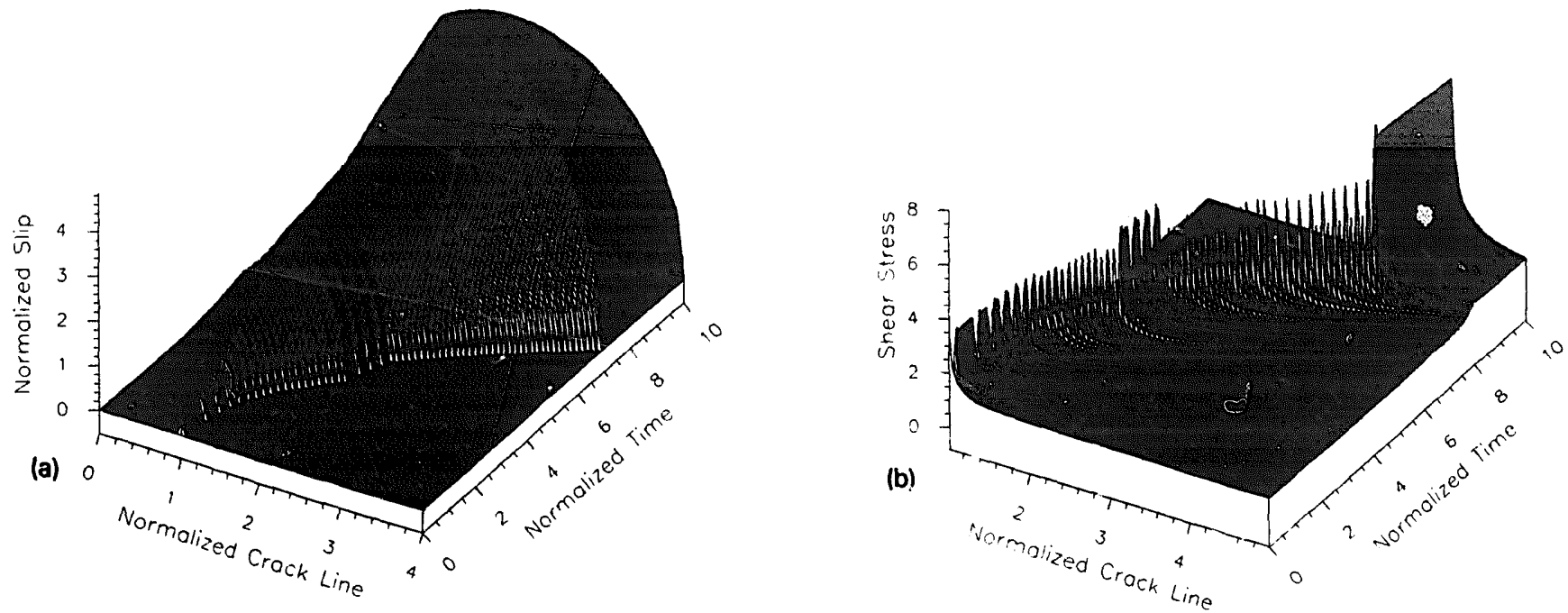

Fig. 10. Slip and stress histories of case 2, Fig. 9 (crack breaking through a barrier).
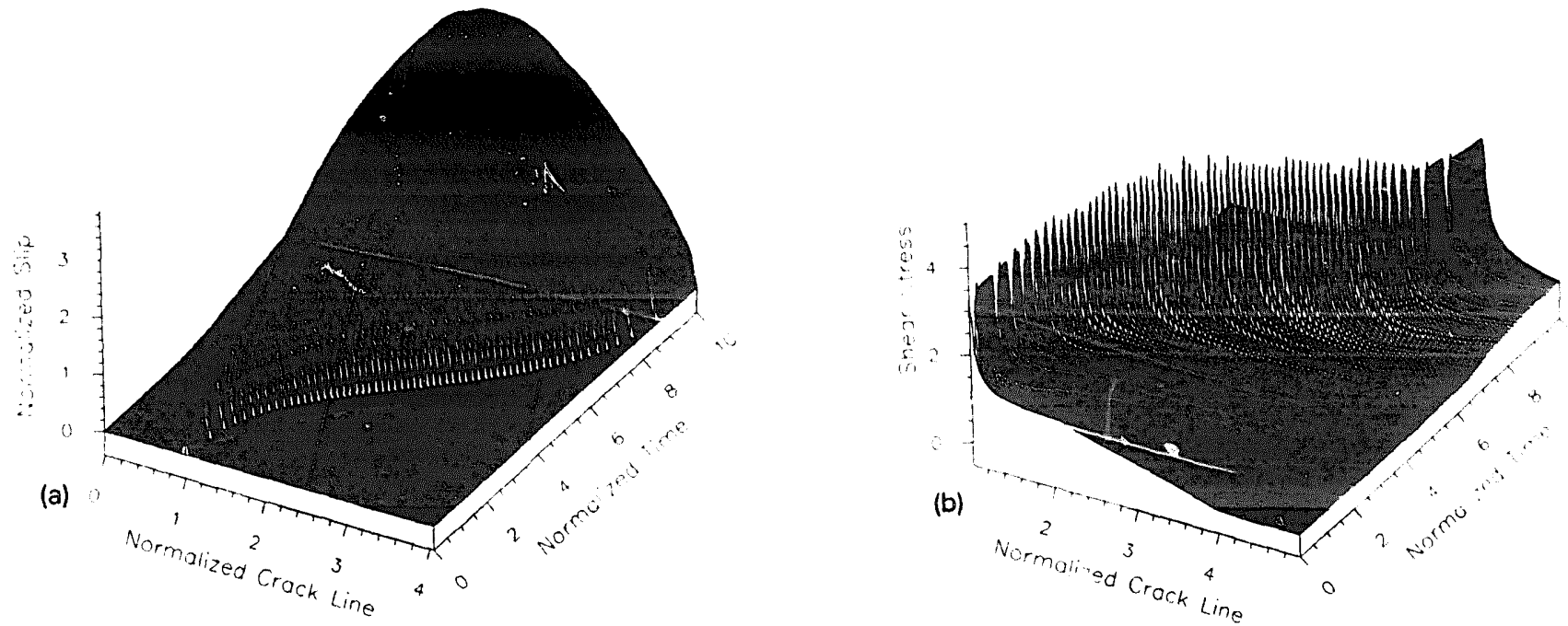

Fig. 11. Slip and stress histories of case 3 in Fig. 9, i.e. of a crack running into a zonc of decreasing ambient stress field.

\subsection{Numerical study of the onset of kinking}

A dynamically propagating crack under antiplane shear may experience kinking (see Section 4.2 ) if $\bar{\sigma}_{93}(\rho, \theta, t)$ is maximized (for fixed $\rho$ ) at a nonzero angle $\theta_{K}(t)$. The numerical determination of $\theta_{K}(t)$ will bo: highly sensitive to the high-frequency disturbances, for they reach the stress calculation points $(\rho, \theta)$ at times which depend on $\hat{\theta}$.

Here the kinking of a crack nucleating from an initially static precrack modelled with 20 elements (case 1 of Fig. 9), is considered. After each time siep, $\bar{\sigma}_{\theta 3}(\rho, 0, t)$ is computed for $\rho=\Delta x / 2$ and $\theta=$ $0^{\circ}, 15^{\circ}, \ldots, 75^{\circ}, 90^{\circ}$. Once $v(t)$ has reached a value of $2 c / 3$, a few kinking attempts were observed, the rupture criterion being satisfied at $\theta=15^{\circ}$. However, it was only when reaching $v(t)=6 c / 7$, after a running distance $l=3.5 L$, that the kinking turned out to be "stable" (in the sense of the procedure described in Section 4). This occurred for $\theta=45^{\circ}$ or $60^{\circ}$ (a choice between these two angles would he based on 
unsignif ant differences in the value of $\left.\bar{\sigma}_{\theta 3}(\rho, \theta, t)\right)$. The same study for a suddenly appearing crack and using 40 elements did not introduce a significant change in these results.

The time elapsed between the instant of kinking and the instant at which (36) is satisfied in front of the kinked element in our numerical calculations suggests that $v(t)$ does not slow down significantly due to kinking, at least at its onset.

No analytical solution is known which exactly corresponds to the kinking problem at hand. The symmetric bifurcation of a running crack was treated by Achenbach [2], whose results were corrected later by Dempsey et al. [20]. Bifurcation was found to occur at $v(t)=0.6 \mathrm{c}$ withi an angle of $90^{\circ}$; immediately after branching, $v(t)$ was extremely low (about $0.02 c$ ), but was rapidly growing.

Experimental results, though done in mode $I$ (see [19] or [20] for references), usually show kinking angles from $10^{\circ}$ to $45^{\circ}$ at $v(t) \approx 0.5 c$ to $0.6 c$. The crack velocity immediately after kinking generally shows little or no change from the pre-branching value. The present numerical results are thus not farther away from what is expected in experimental evidence than the conclusions of the above-mentioned analytical works. However, the kinking velocity seems to be highly overestimated here.

Figure 12 shows how the stress $\sigma_{\theta 3}$ in front of the "running" crack tip typically builds up, from time step to time step after a crack tip jump, in the case of one spatial jump per three time steps (i.e. $v=2 c / 3$ ). The ratios of out-of-plane $(\theta \neq 0)$ to in-plane $(\theta=0)$ stresses show oscillations of magnitude much higher than the small differences to 1 that are expected to occur for them (see Table 1). Since the time-dependent stresses are properly evaluated around a fixed crack (see Fig. 4), the inaccuracies of these ratios are certainly a consequence of the high-frequency perturbations induced by the stepwise numerical crack propagation. This explains, in our opinion, the present unsatisfactory numerical value of the kinking velocity. This situation is not improved when finer meshes are used as long as $\Delta t / \Delta x$ remains constant; moreover, using several values of $\Delta t / \Delta x$ would require too many calculations. This situation, however, is expected to improve considerably once a cohesive zone [28] will be introduced.

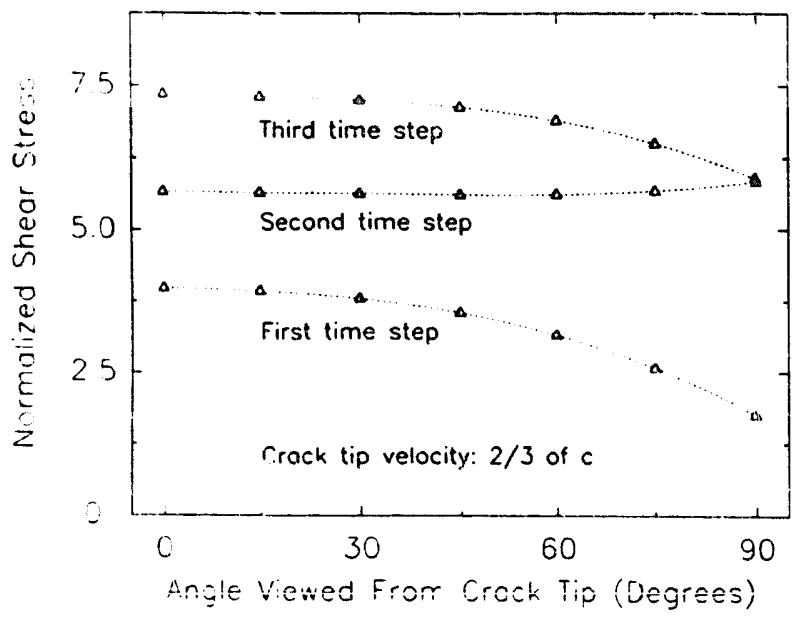

Fig. 12. Shear stress development around the "running" crack tip. from the time step to time step after a crack tip jump. I he ratio $\tau_{H 3}(\theta) / \sigma_{\theta 3}(0)$ does not cievelop smoothly.

We are aware of the fact that the kinking results presented here are tentative. Other criteria, particularly energetic ones, would have to be tested too, but this was beyond the scope of the present work. Furthermore, as pointed out by Freund [23], it might be impossible to establish a realistic branching criterion without taking into account a finite process zone. 


\section{Conclusion}

We have proposed a new boundary integral equation for solving two-dimensional transient dynamical crack problems of general geometry. This method is based on a regularization of the time-domain traction BIE of the crack problem. The singularities of the traction BIE are removed by a technique proposed by Bonnet [8, 9] and implemented here for the particular case of antiplane loading. Special crack tip elements similar to those used by Hirose and Achenback [27] were used in order to improve the stress field resolution near the borders of the crack. Several problems of interest in elastodynamic crack theory were solved with our method and the numerical results obtained were compared to solutions obtained by other numerical methods and with analytical solutions in the few cases where these are available.

Our goal is to develop a stable numerical method for solving dynamical crack-growth problems with a view to applications in seismology and metallurgy. Classical methods like finite differences or finite elements are difficult to apply to the study of crack growth because of their intrinsic numerical dispersion of high frequency elastic waves. Integral equations, on the other hand, give much better results as shown by [5] and [18]. However, the classical formulation used by seismologists, proposed by Das and Aki [17], can only be used to solve problems with axial symmetry, so that only flat collinear cracks may be studied. In seismology, however, the most important problem is understanding the role of crack interaction and geometry in controlling rupture growth. The method proposed here can deal with several cracks of general shape that dynamically interact and is therefore a first step in the direction of studying complex crack growth.

A difficulty that has been only partially solved here is that of the estimation of the stress field directly in front of the rupture front. In our formulation the crack jumps by a grid space whenever a simple stress rupture criterion is satisfied, often attributed to Irwin in the seismological literature. In future we plan to introduce a more realistic slip- or velocity-weakening criterion ([28] or [18]) in order to calculate frartur's energy and establish a criterion based on more appropriate energy dissipation considerations. The same problem arises for the computation of hoop stresses in fr $r$ t of the rupture front in studies of crack branching and kinking. In this case, we believe that results will improve significantiy if we use collocation of the BIE at points closer to the rupture front in the crack-tip element.

\section{Acknowledgments}

The support of the first author by the Swiss National Science Foundation and the free use of the infrastructure at the Laboratoire de Géophysique Interne et Tectonophysique (LGIT), Grenoble, are gratefully acknowledged. All numerical results presented here were obtained using the Cray 2 supercomputer of the Centre de Calcul Vectoriel pour la Recherche, Ecole Polytechnique, Palaiseau, France.

The authors are indebted to Prof. Jean Virieux, of Université de Nice, France, for helpful discussions. The stimuiating atmosphere of mutuai heip at the LGiT has been of great heip for the present work. They also wish to thank the referee for his careful review of this paper and helpful comments.

\section{References}

[1] J.D. Achenbach, "Brittle and ductile extension of a finite crack by a horizontally polarized shear wave", int. J. Eng. Sci. 8, 947 966 (1970).

[2] J.D. Achenbach, "Bifurcation of a running crack in antipiane strain", Int. I Solids d Structures 11, 1301 1314 (1975). 
[3] D.J. Andrews, "Rupture propagation with finite stress in antiplane strain", J. Geophys. Research 81, 3575-3582 (1976).

[4] D.J. Andrews, "Rupture velocity of plane strain shear cracks", J. Geophys. Research 81, 5679-5687 (1976).

[5] D.J. Andrews, "Dynamic plane-strain shear rupture with a slip-weakening friction law calculated by a boundary integral method”, Bull. Seismol. Soc. Am. 75(1) 1-21 (1985).

[6] E. Becache, Résolution par une méthode d'équations intérales d'un problème de diffraction d'ondes élastiques transitories par une fissure, PhD Thesis, Université Paris 6, Paris, France, 1991.

[7] D.E. Beskos (Editor), Boundary Element Methods in Mechanics, Computational Methods in Mechanics, 3, North-Holland, Elsevier, Amsterdam (1987).

[8] M. Bonnet, Méthode des équations intégrales régularisées en élastodynamique tridimensionelle, PhD thesis, Ecole Nationale des Ponts et Chaussées, Paris, France, 1986.

[9] M. Bonnet, "Regularized boundary integral equations for three-dimensional bounded or unbounded elastic bodies containing curved cracks of arbitrary shape under dynamic loading", in: C.A. Brebbia and N.G. Zamani, eds., Boundary Element Techniques: Applications in Engineering, Computational Mechanics Publications, Southampton (1989).

[10] M. Bonnet and H.D. Bui, "Regularization of the displacement and traction BIE for 3D elastodynamics using indirect methods", to appear in: J.H. Kane, G. Maier, N. Tosaka and S.N. Atluri, eds., Advances in Boundary Element Techniques, Springer, Berlin (1992).

[11] M. Bouchon, "Predictability of ground displacement aind velocity near an earthquake fault: an example: the Parkfield carthquake of 1966", J. Geoph. Research 84, 6149-6156 (1979).

[12] C.A. Brebbia, J.C.F. Telles and L.C.Wrobel, Roundary Element Techniques. Theory and Application in Engineering, Springer, Beriin (198.4).

[13] H.D. Bui, "An integral equation method for solving the problem of a plane crack of arbitrary shape", J. Mech. Phys. Solids 25, 29-39 (1977).

[14] R. Burridge, "The numerical solution of certain integrai equations with non-integrable kernels arising in the theory of crack propagation and elastic wave diffraction", Fhilos. Trans. Roy. Soc. London A 265, 353-381 (1969).

[15] D.M. Cole, D.D. Kosloff and J B. Minster, "A numerical boundary integral equation method for elastodynamics I", Bull. Seismol. Soc. Am. 68, 1331-1357 (1978).

[16] T.A. Cruse, Boundary Element Analysis in Computational Fracture Mecriunics, Kluwer Academic Publishers, Dordrecht (1988).

[17] S. Das and K. Aki, "A numerical study of two-uimensional spontaneous rupture propagation", Geophys. J. Roy. Astr. Soc. 50, 643-668 (1977).

[18] S. Das and B.V. Kostrov, Earthquake Source Mechanics, Cambridge University Press, Cambridge (199(1).

[19] J.P. De anpsey and P. Burgers, "Dynamic crack branching in brittle solids", Int. J. Fract. 27 203-213 (1985).

[20] J.P. D mpsey, M.K. Kuo and D.L. Bentley, "Dynamic effects in mode III crack bifurcation", Int. J. Solids \& Strucures 22, 333 $3 ;($; 986$)$.

[21] 2. Liongarra. J.R. Bunch, C.B. Moler and G.B. Stewart, LINPACK Users' Guide, SIAM, Philadelphia (1979).

[22] $A$. Fringen and E.S. Suhubi, Elastodynamics (Vol II Linear Theory), Academic Press, New York (1975).

[23] L. S. Freund, Dynamic Fracture Mechanics, Cambridge University Press, Cambridge (1990).

[24] Guiggiani and A. Gigante, "A gencral algorithm for multidimensional Cauchy principal value integrals in the boundary siement method", to appear in: ASME J. Appl. Mech.

[25] M. Guiggiani, G. Krishnasamy, T.J. Ruldophi and F.J. Rizzo, "A general algorithm for the numerical solution of hypersingular boundary integral equations", to appear in: ASME J. Appl. Mech.

[26] R.A. Harris, R.J. A.rchuleta and S.M. Day, "Fault steps and the rynamic rupture process: $2 \mathrm{D}$ numerical simulations of a spontaneously propagating shear fracture", Geoph. Res. Lett., 18, $893-896$ (1991)

[27] S. Hirose and J.D. Achenbach, "Time-domain boundary element analysis of elastic wave interaction with a crack", int. J. Num. Meth. in Eng. 28, 629-644 (1989).

[28] Y. Ida, "Cohesive force across the tip of a longitudinal shear crack and Griffith's specific surface energy", J. Geophys. Res. 7, 37963805 (1972).

[29] T. Kato, K. Rybicki and K. Kasahara, "Mechanical interaction between neighboring active faults an application to the Atera fault, central Japan". Tectonophysics 114, $181-188$ (1987).

[30] D.L. Karabalis, "A simplified 3-D time-domain BEM for dynamic soil-structure interaction problems", Eng. Anal. with Boundary Elements 8(3) 139-145 (1991).

[31] B.V. Kostrov, "Unsteady propagation of longitudinal shear cracks", J. Appl. Math. Mech. (PMM), 30,1241 1248 (1966).

[32] G. Krishnasamy, F.J. Rizzo and T.J. Rudolphi, "Hypersingular boundary integral equations: their occurrence, interpretation, regularization and computation", to appear in: P.K. Banerjee and S. Kobayashi, eds., Developments in Boundary Element Methods 7: Advanced Dynamic Analysis, Ch. 7.

[33] C.C. Ma and P. Burgers, "Initiation, propagation and kinking of an antiplane crack", ASME J. Appl. Mech. 55, 111-119 (1988).

[34] R. Madariaga, "Dynamics of an expanding circular fault", Bull. Seismol. Soc. Am. 65. 639-666 (1976)

[35] N. Nishimura, Q.C. Guo and S. Kobayashi, "Boundary integral equation method for elastodynamic crack problems", in: Brebbia. Wendland and Kuhn, eds., Boundary Elements IX, Vol. 2, Springer, Berlin (1987). 
[36] F.. Rizzo, D.J. Shippy and M. Rezayat, "A boundary integral equation method for radiation and scattering of elastic waves in three-dimensions", Int. J. Num. Meth. Eng. 2I, 115-129 (1985).

[37] P. Segall and D.D. Pollard, "Mechanics of discontinuous faults", J. Geophys, Res. 85, 4337-4350 (1980).

[38] J. Sladek and V. Sladek, "Three-dimensional curved crack in an elastic body", Int. J. Solids Struct. 19, 425 436 (1983).

[39] I.N. Sneddon, The Use of Integral Transforms, McGraw-Hill, New York (1972).

[40] J. Stoer and R. Bulirsch, Introduction to Numerical Analysis, Springer, Berlin (1980).

[41] H. Tada, P.C. Paris and G.R. Irwin, The Stress Analysis of Cracks Handbook, Hellertown, PA (1973).

[42] A.N. Tikhonov and V.Y. Arsenin, Solutions to Ill-posed Problems, Winston-Wiley, New York (1977).

[43] C.I. Trifu and M. Radulian, "Predicted near-field ground motion for dynamic stress-drop models", PAGEOPH 123, 173-198 (1985).

[44] J. Virieux and R. Madariaga, "Dynamic faulting studied by a finite difference method", Bull. Seismol. Soc. Am. 72(1) 345-369 (1982).

[45] D.J. Wald, D.V. Helmberger and T.H. Heaton, “Analysis $\mathrm{ff}$ the 1989 Loma Prieta earthquake from the inversion of local strong motion and broadband teleseismic data", Abstract, EOS Trans. $71(43) 1454$ (1990).

[46] J. Weaver, "Three-dimensional crack analysis", Int. J. Solids Struct. 13, 321-330 (1977).

[47] G. Yielding, M. Ouyed, G.C.P. King and D. Hatzfeld, "Active tectonics in the Algerian Atlas Mountains - evidence from aftershocks of the 1980 El Asnam earthquake", Geophys. J. Int. 99, 761-788 (1989).

[48] Ch. Zhang, "A novel derivation of non-hypersingular time-domain BIEs for transient elastodynamic crack analysis", Int. $J$. Solids Struct. 28, 267-281 (1991).

[49] Ch. Zhang and J.D. Achenbach, "A new boundary integral equation formulation for elastodynamic and elastostatic crack analysis," Trans. ASME, J. Appl. Mech. 56, 284-290 (1989).

\section{Appendix A. Two-dimensional Green tensors for antiplane problems}

The nonzero components of the impulsive displacement and stress Green's tensor and of the static stress Green's tensor, for the infinite space under antiplane strain, are given by

$$
\begin{aligned}
& \Sigma_{\gamma}(x, y, t-\tau)=-\frac{y_{\gamma}-x_{\gamma}}{2 \pi r}\left\{\frac{\delta(t-\tau-t / c)}{\left((t-\tau)^{2}-r^{2} / c^{2}\right)^{1 / 2}}-\frac{r H(t-\tau-t / c)}{\left((t-\tau)^{2}-r^{2} / c^{2}\right)^{3 / 2}}\right\} \\
& U(x, y, t-\tau)=\frac{1}{2 \pi \mu} \frac{H(t-\tau-r / c)}{\left((t-\tau)^{2}-r^{2} / c^{2}\right)^{1 / 2}} \\
& \Sigma_{\gamma}^{0}(x, y)=-\frac{y_{\gamma}-x_{\gamma}}{2 \pi r^{2}}
\end{aligned}
$$

respectively. From eqs. (47) and (48), the analytic expression of convoldiion integrals which appear $i_{i 1}$ the time-domain BIEs can be derived as follows [22]:

$$
\begin{aligned}
& \int_{0}^{t} \tilde{U}(z, y, t-\tau) \hat{f}(\tau) \mathrm{d} \tau=\frac{H(t-\tau-r / c)}{2 \pi \mu} \int_{r / c}^{t} \frac{f(t-\tau)}{\left(\tau^{2}-r^{2} / c^{2}\right)^{1 / 2}} \mathrm{~d} \tau \\
& \int_{0}^{t} \Sigma_{\gamma}(z, y, t-\tau) f(\tau) \mathrm{d} \tau \\
& \quad=-\frac{y_{\gamma}-x_{\gamma}}{2 \pi r}\left[\frac{1}{r} f(t-r / c)+\frac{\ddot{c}}{c^{2}} \int_{r / c}^{t} \frac{\dot{f}(\hat{\imath}-\tau)}{\left(^{2}-r^{2} / c^{2}\right)^{1 / 2}\left[\tau+\left(\tau^{2}-r^{2} / c^{2}\right)^{1 / 2}\right]} \mathrm{d} \tau\right]
\end{aligned}
$$


If one inserts $f(t)=S(t / \Delta t-m)$ in $(51)$ and $f(t)=\ddot{S}(t / \Delta t-m)$ in (50), the integrals with respect to $\tau$ have the following analytic expressions:

$$
\begin{aligned}
& \int_{r / c}^{i_{n}} \frac{\dot{S}(n-m-\tau / \Delta t)}{\left(\tau^{2}-r^{2} / c^{2}\right)^{1 / 2}\left[\tau+\left(\tau^{2}-r^{2} / c^{2}\right)^{1 / 2}\right]} \mathrm{d} \tau \\
& \quad=\left[\frac{1}{[\tau-r / c]^{+}+r / c+A^{+}(\tau)}\right]_{(m-1) \Delta t}^{m \Delta t}-\left[\frac{1}{[\tau-r / c]^{+}+r / c+A^{+}(\tau)}\right]_{m \Delta t}^{(m+1) \Delta t} \\
& \int_{r / c}^{t_{n}} \frac{\ddot{S}(n-m-\tau / \Delta t)}{\left(\tau^{2}-r^{2} / c^{2}\right)^{1 / 2}} \mathrm{~d} \tau=\left[\frac{1}{A^{+}(\tau)}\right]_{m \Delta t}^{(m+1) \Delta t}-\left[\frac{1}{A^{+}(\tau)}\right]_{(m-1) \Delta t}^{m \Delta t}
\end{aligned}
$$

with $A_{+}(\tau)=\sqrt{\left[\tau^{\left.2-r^{2} / c^{2}\right]^{+}}\right.}$and []$^{+}=\max (, 0)$.

\section{Appendix B. Evaluation of the elementary integrals $H G S$}

In order to evaluate $H G S_{i j}^{0}$ and $H G S_{i j}^{1}$, two auxiliary integrals $I_{i j}$ and $J_{i j}$ are considered:

$$
\begin{aligned}
& I_{i j}=\tau_{\gamma}(x) \int_{E_{i}} \frac{y_{\gamma}-x_{\gamma}}{r^{2}} v^{\prime j}(s)[S(-r / c \Delta t)-1] \mathrm{d} s_{y} \\
& J_{i j}=\tau_{\gamma}(x) \int_{E_{i}} \frac{y_{\gamma}-x_{\gamma}}{r^{2}}\left[v^{\prime j}\left(s_{i}-v^{\prime j}\left(s_{i-1 / 2}\right)\right] \mathrm{d} s_{y} .\right.
\end{aligned}
$$

Since, for $\boldsymbol{x}$ and $y$ both on a straight line,

$$
\tau_{\gamma} \frac{y_{\gamma}-x_{\gamma}}{r^{2}}=\frac{1}{y_{\gamma}-x_{\gamma}}=\frac{1}{s-s_{i-1 / 2}} \text { and } r=\left|s-s_{i-1 / 2}\right|
$$

one has

$$
\begin{aligned}
I_{i j} & =\int_{s_{t-1}}^{s_{i}} \frac{v^{\prime j}(s)}{s-s_{i-1 / 2}}\left[1-\frac{\left|s-s_{i-1 / 2}\right|}{c \Delta t}-1\right] \mathrm{d} s \\
& =-\frac{1}{c \Delta t} \int_{s_{i-1 / 2}}^{s_{i}} v^{\prime j}(s) \mathrm{d} s+\frac{1}{c \Delta t} \int_{s_{i-1}}^{s_{i-1 / 2}} v^{\prime j}(s) \mathrm{d} s .
\end{aligned}
$$

Consideration of all the possible cases for $v^{\prime \prime}(s)$ in $(55),(57)$ leads to:

- $i=2, \ldots, J-1$.

One has $v^{\prime i}(s)=1, v^{\prime i-1}(s)=-1, v^{\prime j}(s)=0(j \neq i-1, i)$. Thus

$$
\begin{aligned}
& I_{i j}=0 \\
& J_{i j}=0
\end{aligned}
$$

the latter being a consequence of property (12) of the basis functions $v^{j}$.

- $i=1$. 
One has $v^{\prime \prime}(s)=(\mathrm{d} / \mathrm{d} s) \sqrt{s / \Delta x}=1 /(2 \sqrt{s \Delta x}), v^{\prime j}(s)=0$ for $j \neq 1$. This

$$
\begin{aligned}
& I_{11}=-\frac{1}{c \Delta t}\left[\sqrt{\frac{s}{\Delta x}}\right]_{\Delta x / 2}^{\Delta x}+\frac{1}{c \Delta t}\left[\sqrt{\frac{s}{\Delta x}}\right]_{0}^{\Delta x / 2}=\frac{1}{c \Delta t}(\sqrt{2}-1) \\
& I_{1 j}=0, \quad j \geqslant 2 .
\end{aligned}
$$

Besides,

$$
v^{\prime \prime}(s)-v^{\prime 1}\left(s_{i-1 / 2}\right)=\frac{1}{2 \Delta x}\left[\sqrt{\frac{\Delta x}{s}}-\sqrt{2}\right]=-\frac{1}{\Delta x}\left[\frac{s-\Delta x / 2}{\sqrt{s \Delta x}+s \sqrt{2}}\right]
$$

thus

$$
\begin{aligned}
J_{11} & =-\frac{1}{\Delta x} \int_{0}^{\Delta x} \frac{s-\Delta x / 2}{\sqrt{s \Delta x}+s \sqrt{2}} \frac{\mathrm{d} s}{s-s_{1 / 2}} \\
& =-\frac{1}{\Delta x} \int_{0}^{\Delta x} \frac{\mathrm{d} s}{\sqrt{s \Delta x}+s \sqrt{2}}=-\frac{2}{\Delta x} \int_{0}^{\sqrt{\Delta x}} \frac{\mathrm{d} u}{\sqrt{\Delta x}+u \sqrt{2}}, \quad s=u^{2} \\
& =-\frac{\sqrt{2}}{\Delta x} \log (1+\sqrt{2}) \\
J_{1 j} & =0, \quad j \geqslant 2 .
\end{aligned}
$$

- $i=J$.

A similar calculation gives

$$
\begin{array}{ll}
I_{J,(J-1)}=\frac{1}{c \Delta t}(1-\sqrt{2}), & I_{J, j}=0, \quad j \leqslant J-2 \\
J_{J,(J-1)}=-\frac{\sqrt{2}}{\Delta x} \log (1+\sqrt{2}), & J_{J, j}=0, \quad j \leqslant J-2 .
\end{array}
$$

Finally, from eqs. (66) and (68):

$$
\begin{aligned}
H G S_{i j}^{0} & =\tau_{\gamma}(x) \int_{E_{i}} \frac{y_{\gamma}-x_{\gamma}}{r^{2}}\left[\left[v_{j}^{\prime}(s)-v_{j}^{\prime}\left(s_{i-1 / 2}\right)\right]+v^{j}(s)[S(-r / c \Delta t)-1]\right] \mathrm{d} s_{y} \\
& =I_{i j}+J_{i j} \\
H G S_{i j}^{1} & =-\tau_{\gamma}(x) \int_{E_{i}}^{y_{\gamma}-x_{\gamma}} \frac{y^{2}}{r^{2}}(s)[S(-r / c \Delta t)-1] \mathrm{d} s_{y} \\
& =-I_{i j} .
\end{aligned}
$$


Collecting (58) to (69) gives the result

$$
\begin{array}{ll}
H G S_{11}^{0}=\frac{1}{c \Delta t}(\sqrt{2}-1)-\frac{\sqrt{2}}{\Delta x} \log (1+\sqrt{2}) & \\
H G S_{1 j}^{0}=0, & j=2, \ldots, J \\
H G S_{i j}^{0}=0, & i=2, \ldots, J-1 \\
H G S_{J,(J-1)}^{0}=\frac{1}{c \Delta t}(1-\sqrt{2})-\frac{\sqrt{2}}{\Delta x} \log (1+\sqrt{2}) & j=2, \ldots, J \\
H G S_{J, j}^{0}=0, & \\
H G S_{11}^{1}=\frac{\sqrt{2}}{\Delta x} \log (1+\sqrt{2}) & \\
H G S_{J,(J-1)}^{1}=\frac{\sqrt{2}}{\Delta x} \log (1+\sqrt{2}) & (i, j) \neq c(1,1),(J, J-1) . \\
H G S_{i, j}^{1}=0, &
\end{array}
$$

